\title{
Comparative Metabolomics Analysis of Juvenile Takifugu obscurus serum and Liver Samples after Exposure to Tributyltin Chloride
}

\author{
Dong-Po Xu ${ }^{1,2 *}$, Di-An Fang ${ }^{1,2}$, Shu-Lun Jiang ${ }^{2}$, Chang-Sheng Zhao ${ }^{1}$ \\ ${ }^{1}$ Freshwater Fisheries Research Center, Chinese Academy of Fishery Sciences, Wuxi, Jiangsu 214081, China \\ ${ }^{2}$ Wuxi Fisheries College, Nanjing Agricultural University, Wuxi, Jiangsu 214081, China
}

Received: 27 July 2020

Accepted: 19 November 2020

\begin{abstract}
Tributyltin chloride (TBT-Cl), one of the most representative chemical compounds of tributyltin (TBT), is a threat to aquatic organisms due to evidence of fatal toxicity, which has attracted much interest in the scientific community. We studied the effects of acute TBT-Cl exposure on the juvenile Takifugu obscurus metabolism by investigating the metabolite contents in the serum and liver at three TBT-Cl concentrations and in a control group using the LC-MS technology. The metabolites that showed different levels in the serum and liver under different TBT-C1 concentrations were primarily related to sphingolipid metabolism, phosphoglyceride metabolism, and the biosynthesis of unsaturated fatty acids. Moreover, we found that TBT-Cl exposure increased the concentration of lysophosphatidylcholine and prostaglandin $\mathrm{E}_{2}$, resulting in higher detoxification capacity and inhibiting inflammation. These results may explain the higher tolerance of juvenile T. obscurus to TBT-Cl compared with other fish species. Overall, the present study contributes new information of the influences of TBT-Cl on juvenile T. obscurus metabolism.
\end{abstract}

Keywords: Takifugu obscures, TBT-Cl exposure, metabolic pathway

\section{Introduction}

Tributyltin chloride (TBT-Cl), a widely used biocide in antifouling ship paints, is a highly toxic organotin compound that acts as a powerful endocrine disruptor, obesogenic, immunotoxic stimulant, and neurotoxic stimulant [1]. As an obesogen, TBT-Cl alters PPARg and RXRa coregulatory complexes on target genes [2], causing metabolic and reproductive dysfunction.

*e-mail: xudp@ffrc.cn
Oligomycin-sensitive Mg-ATPase is a known target of TBT toxicity in mammals and invertebrates [3-5]. At high doses, TBT inhibits the induction of cytochrome $\mathrm{P} 450 \mathrm{~s}$ in the channel catfish Ictalurus punctatus, and in rodents [6]. Furthermore, TBT inhibits steroidogenesis by reducing steroidogenic enzyme expression in interstitial Leydig cells [7]. Due to its toxicological effects on immunity, nervous system, development, and reproduction in both aquatic organisms and mammals, TBT has been globally banned from the beginning of the $21^{\text {st }}$ century [8-10]; however, high levels of TBT residue were still detected in in marine and freshwater environments, for example, $0.5 \mathrm{ng} / \mathrm{L}$ to $977 \mathrm{ng} / \mathrm{L}$ of tin 
in water samples in mainland of China [11, 12], which may endanger aquatic ecosystems.

Takifugu obscurus, commonly known as the river pufferfish, inhabits the East China Sea and the Yellow Sea, and has considerable economic values due to its high nutritional quality [13]. However, its wild resource has suddenly declined in recent years due to overfishing and water contamination; it shows a high adaptive and resistant capacity against environmental stress factors, including TBT exposure [14]. Transcriptome sequencing analysis has been conducted to study the differential mRNA transcription of $T$. obscurus in response to treatments with different concentrations of TBT. The results indicated that $T$. obscurus regulated the immune function to resist the adverse effect of TBT-Cl through mediating HSP90b1 [15]. However, the responses of juvenile T. obscurus to acute $\mathrm{TBT}-\mathrm{Cl}$ exposure at the metabolic level have not yet been reported.

Metabolomics, which originated from metabolic profiling, is a discipline that studies trace amounts of metabolites in living organisms. In this study, comprehensive comparisons and analyses of serum and liver samples between three acute TBT-Cl treatment groups and a control group were conducted using the LC-MS technique. This research provides new understandings of how TBT-Cl exposure disturbs metabolic pathways in T. obscurus.

\section{Materials and Methods}

\section{Reagents, Animals, and Water}

TBT-Cl (98\% purity) was obtained from Dr. Ehrenstorfer, Germany. Juvenile T. obscurus specimens (two months old, average length of $10 \pm 1.5 \mathrm{~cm}$, average weight of $25.1 \pm 2.23 \mathrm{~g}$ ) were obtained from Freshwater Fisheries Research Center (FFRC, Wuxi, China). According to Li et al. [16], Irerhievwie and Akpoghelie [17], juvenile fish are more sensitive to acute toxicant exposure than adults. The fish were cultivated in 100 L cylindrical opaque polypropylene aquaria and fed with commercial feed twice a day at regular intervals. Animals were chosen randomly for experimental treatments after at least 7 days of acclimation. Tap water was aerated for one week before using as the culture media. The temperature, dissolved oxygen, and $\mathrm{pH}$ were kept at $30 \pm 4^{\circ} \mathrm{C}, 6.9 \pm 0.1 \mathrm{mg} / \mathrm{L}$, and $7.8 \pm 0.05$, respectively, which meet the China fishery water quality standard (GB11607-89).

\section{Acute Toxicity Exposure}

The $96 \mathrm{~h} \mathrm{LC50}$ of TBT-Cl to juvenile T. obscurus was $19.62 \mu \mathrm{g} / \mathrm{L}$. In the present study, four groups (A, B, $\mathrm{C}$, and $\mathrm{Z}$ ) of healthy fish were set and exposed to three concentrations of TBT-Cl (A: 10\% 96 h-LC50, B: 20\% 96 h-LC50, and C: 50\% 96 h-LC50) and DMSO solution $\left(\mathrm{V}_{\text {DMSO }}: \mathrm{V}_{\text {water }}=1: 100\right)$. Each treatment included ten fish and repeated three times independently. After $96 \mathrm{~h}$ exposure, four fish from each subgroup were randomly chosen and placed in ice trays. Fish were quickly dissected, and the livers and blood samples were obtained. The blood samples were placed in a sodium heparin centrifuge tube, stood still for $20 \mathrm{~min}$, and then centrifuged at $3500 \mathrm{rpm}$ for 10 minutes at $4^{\circ} \mathrm{C}$. The serum and livers were frozen immediately using liquid nitrogen, and then stored at $-80^{\circ} \mathrm{C}$ for further analysis. All experiments strictly followed the Guide for the Care and Use of Laboratory Animals in FFRC.

\section{Serum and Liver Sample Collection}

For serum, $100 \mu \mathrm{L}$ of each sample mixed with $300 \mu \mathrm{L}$ of methanol ( to precipitate proteins, and 2-chlorophenylalanine $\left(2.9 \mathrm{mg} \cdot \mathrm{mL}^{-1}\right)$ was added as the internal standard. After vortexing for 30 seconds, the mixture was centrifuged at $12,000 \mathrm{rpm}$ for 15 minutes at $4^{\circ} \mathrm{C}$, and $200 \mu \mathrm{L}$ of the supernatant was transferred to injection vials for LC-MS analysis. For liver tissues, $50 \mathrm{mg}$ of each sample were weighed and mixed with $800 \mu \mathrm{L}$ of methanol and $10 \mu \mathrm{L}$ of 2-chlorophenylalanine $\left(2.9 \mathrm{mg} \cdot \mathrm{mL}^{-1}\right)$. The mixtures were homogenized using a tissue grinder at $65 \mathrm{~Hz}$ for $90 \mathrm{~s}$. The samples were then vortexed for 30 seconds and centrifuged at $12,000 \mathrm{rpm}$ for 15 minutes at $4^{\circ} \mathrm{C}$, and $200 \mu \mathrm{L}$ of the supernatant was transferred to injection vials for detection.

\section{LC-QTOF MS Analysis}

An Agilent 1290 Infinity LC coupled with 6530 UHD and Accurate-Mass Q-TOF/MS were used for analysis. All samples were analyzed using a $2.1 \mathrm{~mm} \times 100 \mathrm{~mm}$ Agilent C18 column $(1.8 \mu \mathrm{m})$, which was maintained at $40^{\circ} \mathrm{C}$ and eluted with a multistep gradient of mobile phase A (water, $0.1 \%$ formic acid) and B (acetonitrile, $0.1 \%$ formic acid) over the course of $15 \mathrm{~min}$ at $0.35 \mathrm{~mL} / \mathrm{min}$. The gradient started with a $1 \mathrm{~min}$ isocratic step at $5 \% \mathrm{~B}$, which was increased to $20 \% \mathrm{~B}$ over the next $5 \mathrm{~min}$, and to $50 \%$ B within 9 min. Finally, the gradient was set to $95 \%$ B for $4 \mathrm{~min}$ and was kept isocratic for another $2 \mathrm{~min}$. The injection volume was $4 \mu \mathrm{L}$ and the maximum column pressure was set at 800 bar.

Mass spectrometric data were collected in both positive and negative ESI modes with a mass range of $50-1000 \mathrm{~m} / \mathrm{z}$. The scan duration was $0.03 \mathrm{~s}$ in centroid mode with an interscan time of $0.02 \mathrm{~s}$. The source temperature was $100^{\circ} \mathrm{C}$ with nitrogen at $50 \mathrm{~L} / \mathrm{h}$ as the cone gas, and the extraction cone voltage was $4 \mathrm{~V}$. The lock mass calibration was applied using a solution of leucine encephalin, and its observed molecular weights in positive and negative modes were 556.2771 $\mathrm{Da}$ and 554.2615 Da, respectively. Other parameters at the $\mathrm{ESI}^{+}$mode were set as: capillary voltage of $4 \mathrm{kV}$, sampling cone voltage of $35 \mathrm{~V}$, and desolvation temperature of $350^{\circ} \mathrm{C}$ at $600 \mathrm{~L} / \mathrm{h}$. At the ESI mode, 
parameters were set as: capillary voltage of $3.5 \mathrm{kV}$, sampling cone voltage of $50 \mathrm{~V}$, and desolvation temperature of $300^{\circ} \mathrm{C}$ at $700 \mathrm{~L} / \mathrm{h}$. External calibration of the instrument was performed before each batch of analyses.

\section{Data Analysis}

Raw data were processed using the Mass Profiler (Agilent) software for feature extraction, grouping, and alignment. The detailed parameters, including retention time, mass, sample number, and intensity were then converted to excel files and further processed with SIMCA-P 13.0 (Umetrics AB, Umea, Sweden) for chemometric analysis. PCA plots were constructed to show the differences in metabolites among groups. We used OPLS-DA to locate potential markers contributing to group discrimination based on the $t$-test and the Mann-Whitney $U$-test. The S-plots were generated using the SIMCA-P software. Metabolite annotation was performed manually by comparing the intrinsic parameters, including mass accuracy (with less than $5 \mathrm{ppm}$ error), isotopic distribution, fragments, and retention time with the external Metlin and MassBank databases. R version 3.4 .4 (http://www.R-project.org/) was used for data processing and graphic drawing.

\section{Results}

\section{Comparison of Total Ion Chromatograms of Metabolites in Serum and Liver}

At either positive or negative modes, TIC chromatograms of serum and liver samples showed distinct differences between TBT treatments and the control (Fig. 1). Furthermore, there were observable differences between groups treated with different doses of TBT-Cl. These results suggested notable impacts of TBT-Cl on the metabolic status of juvenile T. obscurus.

\section{Chemometric Analysis of the Metabolites in Serum and Liver Samples}

A total of 812 features were extracted using the Mass Profiler (Agilent) in the serum samples of juvenile $T$. obscurus at positive ion mode, and 554 features were detected at the negative ion mode. Before

\section{Serum samples}

(+)
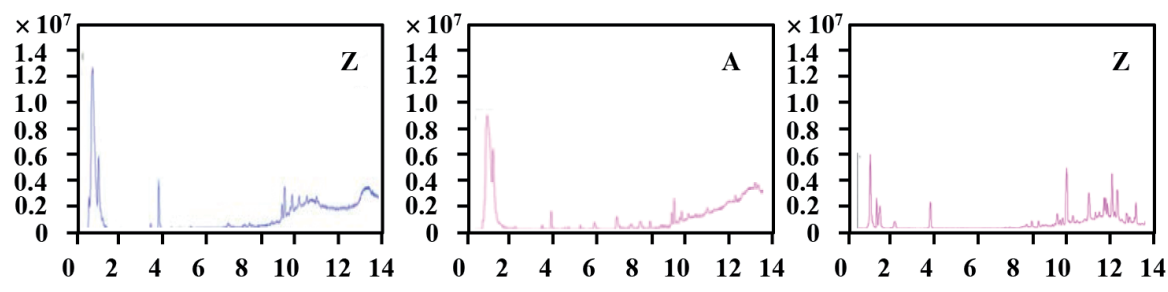

\section{Liver samples}
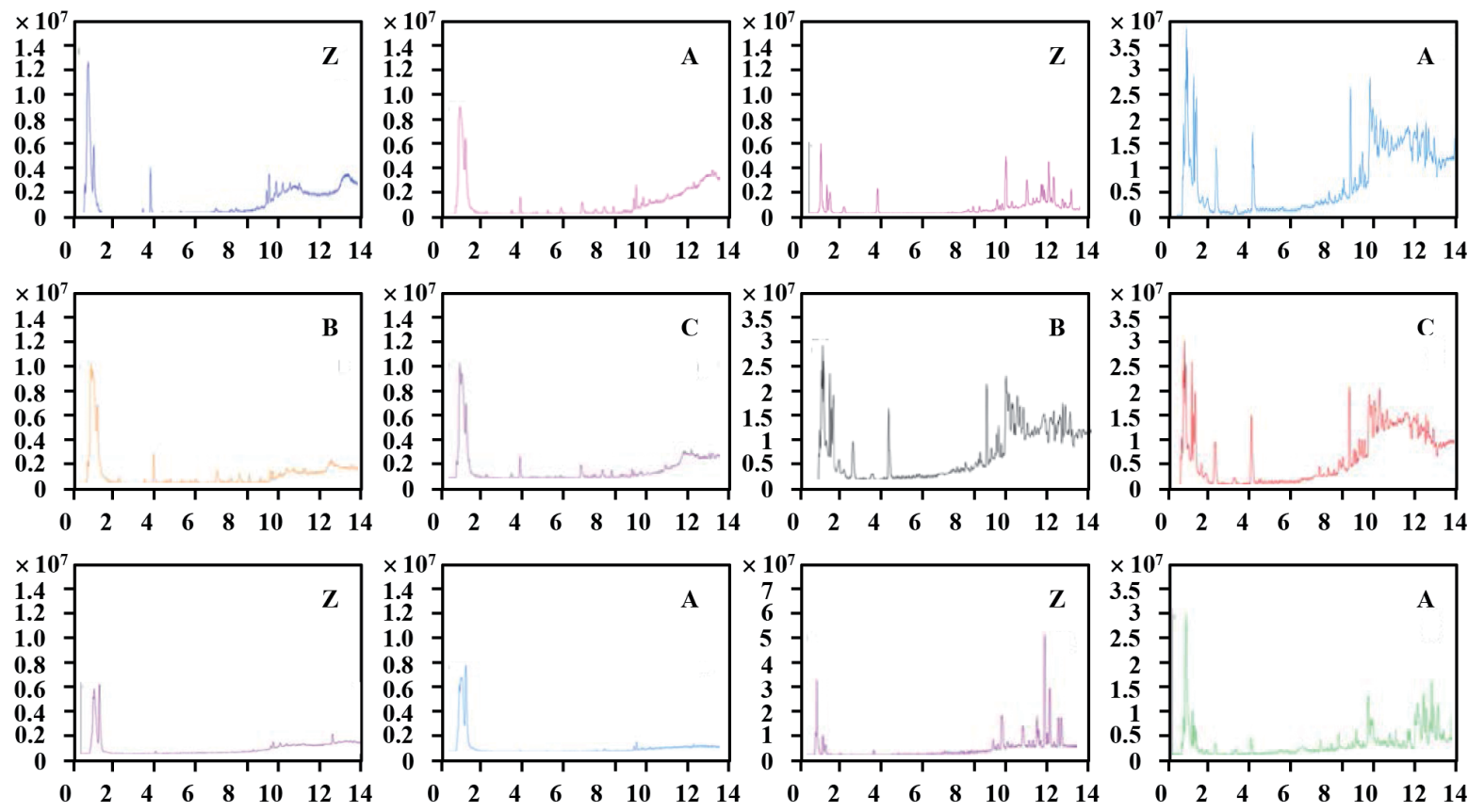

$(-)$
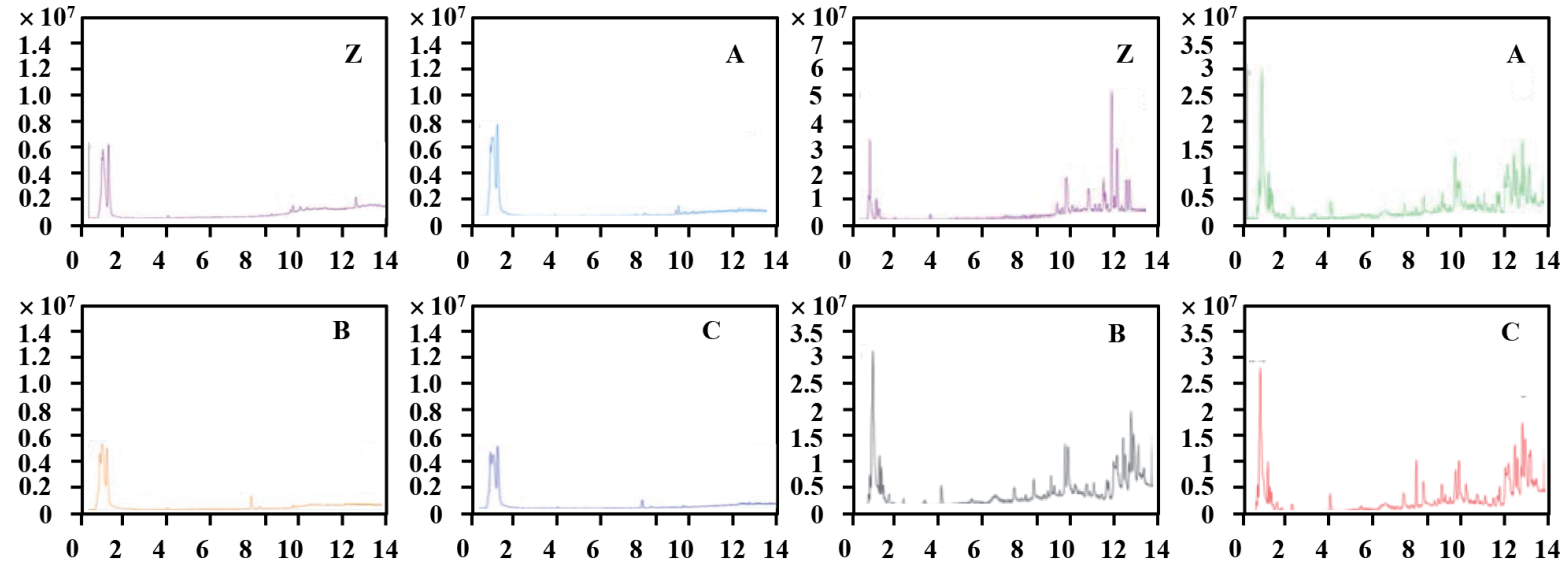

Fig. 1. The TICs of serum and liver samples from four treats $(\mathrm{Z}, \mathrm{A}, \mathrm{B}, \mathrm{C})$ under positive and negative modes. Four groups were designed according to the concentrations of TBT-Cl exposure (Z: DMSO solution, A: $10 \% 96 \mathrm{~h}-\mathrm{LC}_{50}, \mathrm{~B}: 20 \% 96 \mathrm{~h}-\mathrm{LC}_{50}$, and C: $50 \% 96 \mathrm{~h}-\mathrm{LC}_{50}$ ). The serum and liver samples were analyzed with LC-QTOF MS and the TICs under positive and negative modes were shown. 
formal analysis, these data were normalized using the SMICA-P software to better align the variables, including the digital features and scales (such as mean and standard deviation), for more intuitive and reliable results.

Principal component analysis (PCA) was performed for groups A, B, C, and $\mathrm{Z}$ in either positive or negative modes. The PCA model parameters for each group comparison are shown in Table S1, and the PCA score plot appears in Fig. S1. In the score plot, the abscissa and ordinate represent the first and second principal component scores, respectively. According to the data from Table S1, the $\mathrm{R}^{2} \mathrm{X}$ values of the three groups were all greater than 0.55 at both positive and negative modes, indicating an interpretable PCA model and reliable experimental results. According to the PCA score plot (Fig. S1), the comparisons between each treatment group and the control group were significantly distinguished in the top two principal components, suggesting distinct metabolic differences in juvenile $T$. obscurus metabolites between acute TBT-Cl treatments and the control.

OPLS-DA was used to establish a model for statistical analysis of the treatment groups and the control group. According to the OPLS-DA model parameters (Table S2), the $\mathrm{R}^{2} \mathrm{Y}$ values of the three groups were greater than 0.97 in both positive and negative modes, indicating that the model was interpretable. The $\mathrm{Q}^{2}$ values were greater than 0.91, indicating that the model was predictable. As shown in Fig. 2, scattered points were concentrated on both sides of the $\mathrm{t}[1]$ axis, and the distributions of the TBT-Cl treated groups and the control sample were significantly different, indicating good discrimination.

Similarly, a total of 1,391 features were detected in the liver samples of juvenile T. obscurus under positive ion mode, and 1,116 features were detected under negative ion mode. The PCA model parameters of each group are shown in Table S3, and the PCA score plot is shown in Fig. S2. According to the results, the $\mathrm{R}^{2} \mathrm{X}$ values of the three groups were all greater than 0.58 at both positive and negative modes, indicating a high reliability. Moreover, the three comparisons between each treatment group and the control group had significantly different top two principal components at positive and negative modes. These results indicated that the metabolite levels in the TBT-Cl-treated juvenile T. obscurus liver were significantly different from those in the control group.

In order to identify the metabolites leading to these significant differences, OPLS-DA analysis of liver metabolites were conducted to statistically analyze the data of each treatment group and the control group. As shown in Table S4, the $\mathrm{R}^{2} \mathrm{Y}$ values of the three groups were greater than 0.99 in both positive and negative modes, indicating reliable and interpretable results, and the $\mathrm{Q}^{2}$ value was greater than 0.99 , demonstrating a strong predictability of the model. Meanwhile, the OPLS-DA scores in the positive and negative modes showed that the scattered points were concentrated on both sides of the $t[1]$ axis (Fig. 3). The differences between the TBT-Cl-treated and the control liver samples were significant. Therefore, the metabolite levels in each treatment group were significantly different from those in the control group.

\section{Identification of Differential Metabolites in Serum Samples}

Based on the identification results of the OPLS-DA model, a total of 87 differential metabolites were screened at the positive mode (Table S5). Of these metabolites, 40 were upregulated in at least one TBT-Cl treatment in comparison to the control, and 25 of 40 (including arginine succinic acid, 3-methylxanthine, and 4-heptyloxyphenol) were observed in all the three TBT-Cl groups. Conversely, 47 metabolites were downregulated in at least one TBT-Cl treatment, with 17 of 47 (including linoleic acid, L-arginine, and glucoheptanoic acid) being observed in all the three TBT-Cl groups. A total of 34 differential metabolites were screened at the negative mode (Table S6). Among them, 17 were upregulated in at least one TBT-Cl treatments, and 4 of 17 differential metabolites (including Na-acetyl-L-glutamine, L-homotyrosine, and L-aniline) were detected in all the three treatment groups. The other 17 differential metabolites screened at the negative mode were downregulated in TBT-Cl treatments, with 8 of them (such as enoic acid, oleic acid, and linoleic acid) being found in all the three TBT-Cl treatment groups. These differential metabolites mainly belong to phosphoglycerides, unsaturated fatty acids, amino acids, fatty amides, and carnitines (Fig. S3-5).

The identified differential metabolites were analyzed against the KEGG database using zebrafish as the reference to determine the corresponding metabolic pathways. At the positive mode, the main differential metabolic pathways included sphingolipid, glycerophospholipid, alanine, aspartic acid, and glutamate metabolisms. At the negative mode, the differential metabolites were mainly related to biosynthesis of phenylalanine, tyrosine, tryptophan, unsaturated fatty acids, phenylalanine, ubiquinone, linoleic acid, and aminoacyl tRNA.

For liver samples, the OPLS-DA analysis screened 92 differential metabolites at the positive mode (Table S7). Of them, 66 were upregulated in at least one TBT-Cl treatment compared with the control, and 45 of 66 (including adenosine, ADP-Ribosyl-L-arginine, and PE [18: 3 (6Z, 9Z, 12Z)/P-16: 0]) were found in all the three TBT-Cl treatments. Meanwhile, 26 differential metabolites were downregulated in at least one TBT-Cl treatment, with 21 of them (including arachidonic acid, diethylphosphate, and tocopheronic acid) being found in all the three TBT-Cl groups. A total of 71 differential metabolites were screened at the negative mode (Table S8), 54 of which were upregulated in at least 


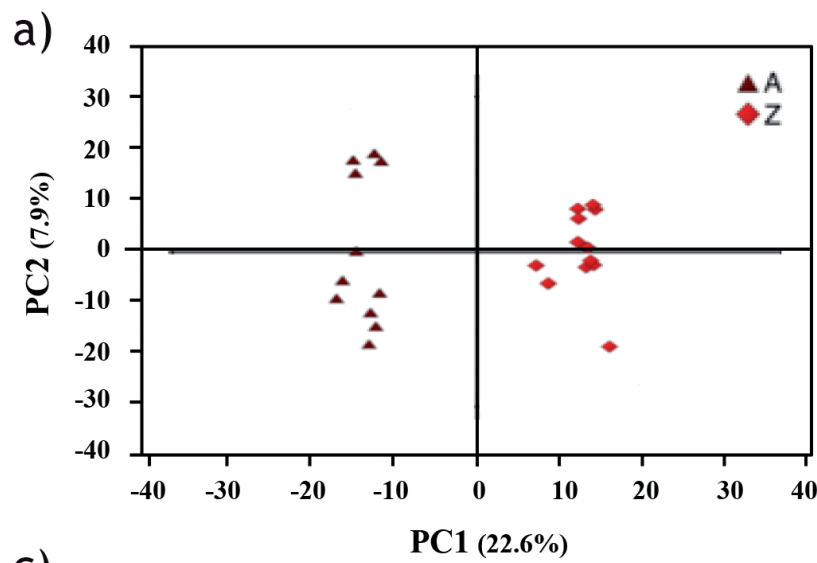

C)
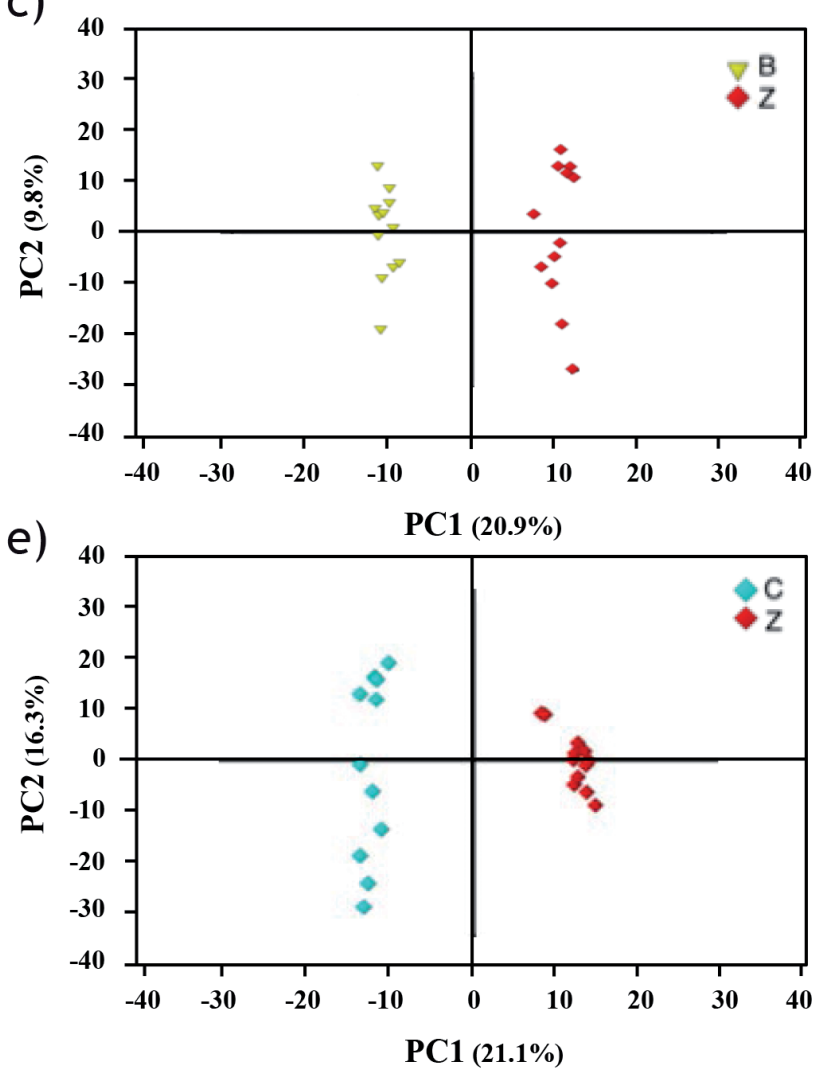

b)

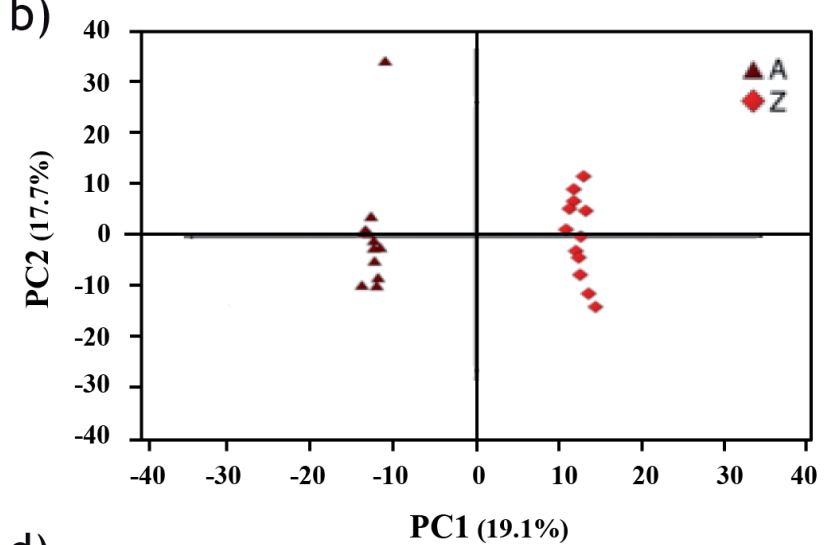

d)
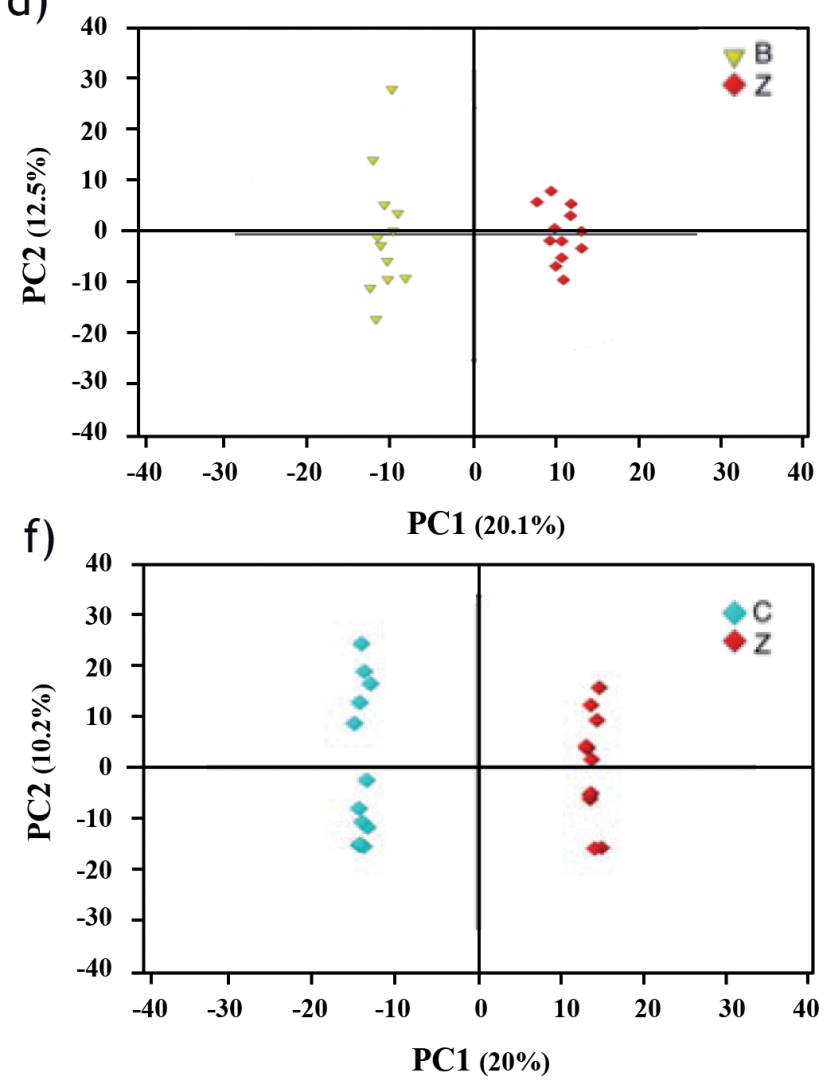

Fig. 2. The OPLS-DA score plots of serum samples A-Z (A: ESI $\left.{ }^{+}, \mathrm{B}: \mathrm{ESI}{ }^{-}\right), \mathrm{B}-\mathrm{Z}\left(\mathrm{C}: \mathrm{ESI}^{+}, \mathrm{D}: \mathrm{ESI}^{-}\right)$and C-Z (E: ESI ${ }^{+}, \mathrm{F}$ : ESI-). a), c) and e). The scoring plot of serum samples A, B, C and Z by comparing the contents of a total of 812 features extracted in positive ion mode. PC1 and PC2 of A-Z, B-Z and C-Z described $22.6 \%$ and $7.9 \%, 20.9 \%$ and $9.8 \%, 21.1$ and $16.3 \%$ of total variability, respectively. b), d) and f). The scoring plot of serum samples A, B, C and Z by comparing the contents of a total of 554 features extracted in negative ion mode. PC1 and PC2 of A-Z, B-Z and C-Z described $19.1 \%$ and $17.7 \%, 20.1 \%$ and $12.5 \%, 20 \%$ and $10.2 \%$ of total variability, respectively.

one TBT-Cl treatment, including arsenous acid, DG (15: 0/16: 0/0: 0), PS (16: 0/16: 0), and 30 of 54 were found in all the three TBT-Cl groups. The other 17 metabolites were downregulated, 16 of which, including palmitic acid, oleic acid, and linoleic acid, were found in all three groups. These differential metabolites were related to phosphoglycerides, unsaturated fatty acids, amino acids, purines, and pyrimidines. (Fig. S6-8).

KEGG analysis revealed that these differential metabolites at the positive mode were mainly related to phosphoglyceride, arginine, proline, arachidonic acid, and linoleic acid, while the differential metabolites at the negative mode were mainly related to phosphate glyceride, unsaturated fatty acid, and linoleic acid.

\section{Effect of TBT-Cl on Metabolic Pathways}

According to the differential metabolites, the relative content of phospholipids (including $16 \mathrm{PCs}$, 13 PEs, and 1 PS) involved in glyceryl phosphate metabolism in the liver significantly increased in the TBT-Cl treated group, except PE [18:1 (11Z)/22:6 
(4Z, 7Z, 10Z, 13Z, 16Z, 19Z)], PC [18:2 (9Z, 12Z)/22:6 (4Z, 7Z, 10Z, 13Z, 16Z, 19Z)], PC (15:0/16:1(9Z)], and PC [14:0/22:5(4Z, 7Z, 10Z, 13Z, 16Z)] (Fig. 4 and Fig. 5). In addition, the relative contents of $1 \mathrm{PG}$ and 5 PIs in the TBT-Cl treatment groups also significantly increased, which indicates that exposure to TBT-Cl stimulated the synthesis of phospholipids in juvenile T. obscurus. Analysis of the differential serum metabolites also showed that 7 PSs, 2 PCs, and 1 PA in the TBT-Cl treatment groups increased significantly, confirming that juvenile $T$. obscurus could accelerate the transportation of phosphoglycerides to resist TBT-Cl toxicity. Additionally, the levels of acetylcholine in the serum samples were significantly lower in the three TBT-Cl treatment groups than those in the control group.

TBT-Cl exposure also affected the metabolism of sphingolipids. As shown in Fig. 6, the serum sphingomyelin content in the treatments A, B, and $\mathrm{C}$ increased 2.69, 2.42, and 2.49 times, respectively, compared with the control, indicating that more sphingomyelin was synthesized after TBT-Cl exposure.
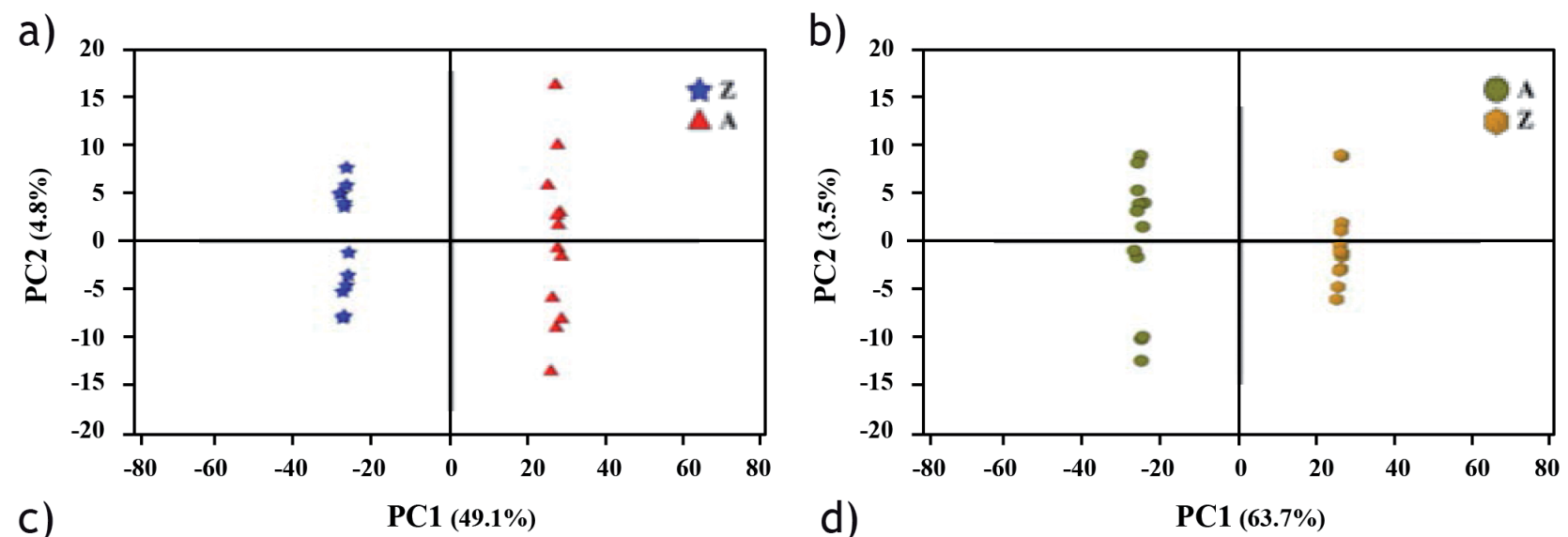

c)

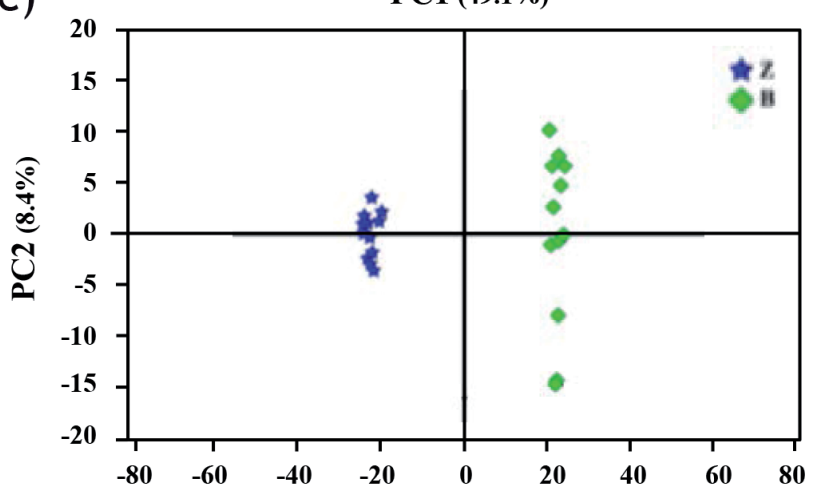

e)
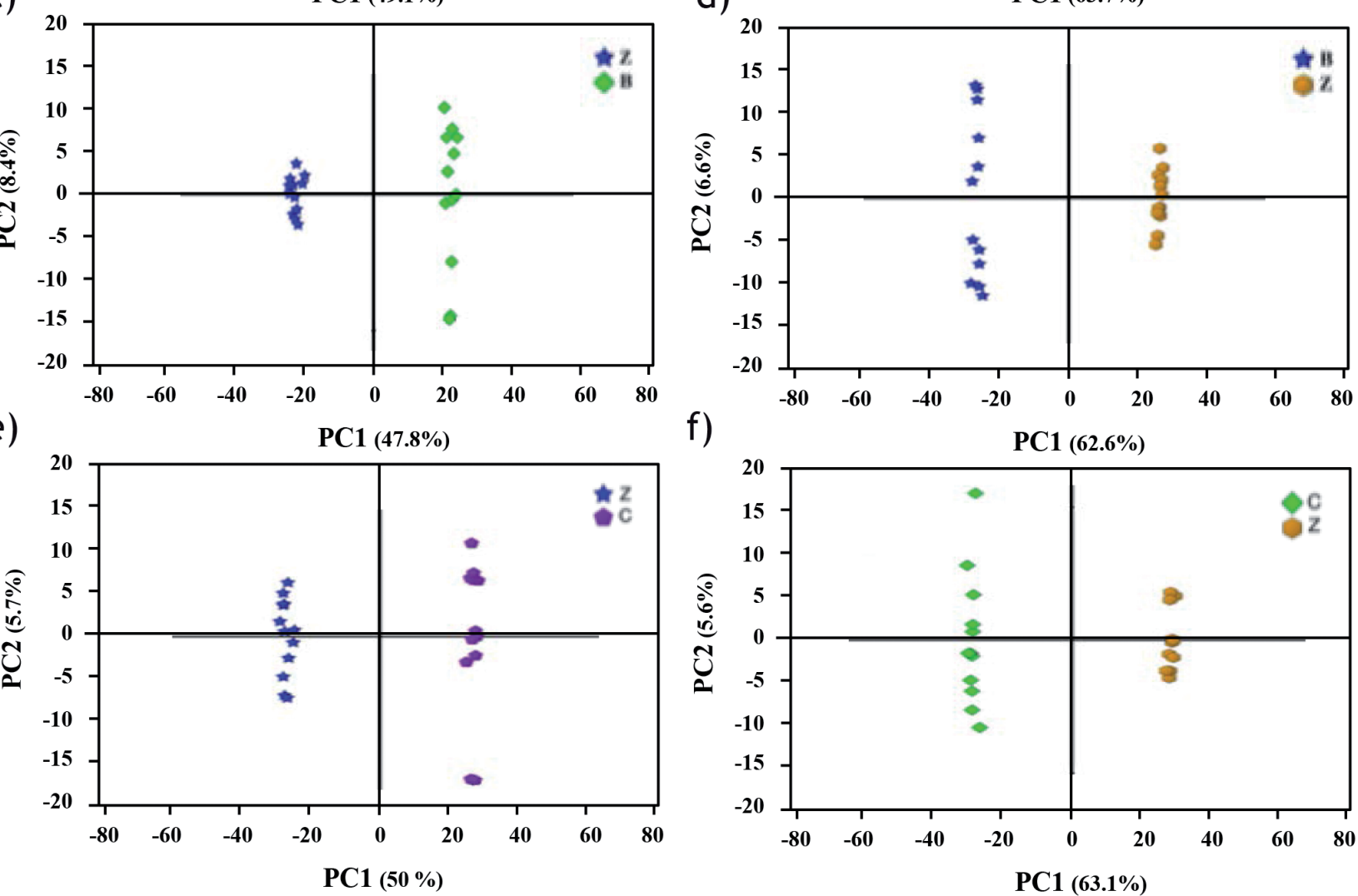

f)

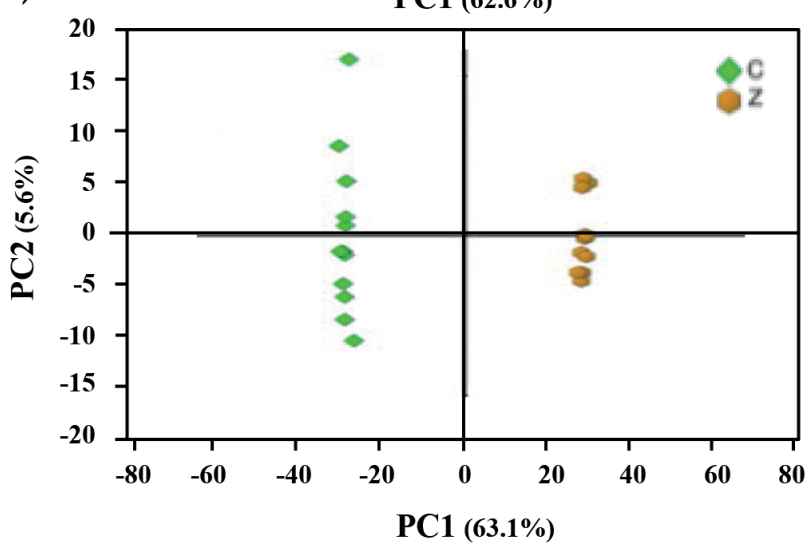

Fig. 3. The OPLS-DA score plots of liver samples A-Z (A: ESI ${ }^{+}$, B: ESI-), B-Z (C: ESI $\left.{ }^{+}, \mathrm{D}: \mathrm{ESI}^{-}\right)$and C-Z (E: ESI', F: ESI-). a), c) and e). The scoring plot of serum samples $\mathrm{A}, \mathrm{B}, \mathrm{C}$ and $\mathrm{Z}$ by comparing the contents of a total of 1,391 features extracted in positive ion mode. PC1 and PC2 of A-Z, B-Z and C-Z described $49.1 \%$ and $4.8 \%, 47.8 \%$ and $8.4 \%, 50$ and $5.7 \%$ of total variability, respectively. b), d) and e). The scoring plot of serum samples A, B, C and Z by comparing the contents of a total of 1,116 features extracted in negative ion mode. PC1 and PC2 of A-Z, B-Z and C-Z described $63.7 \%$ and $3.5 \%, 62.6 \%$ and $6.6 \%, 63.1 \%$ and $5.6 \%$ of total variability, respectively. 

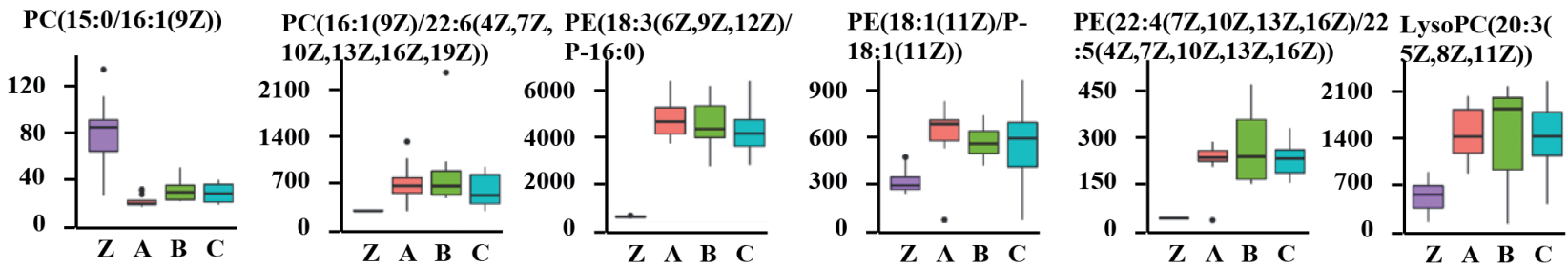

PC(14:0/20:5(5Z,8Z PC(18:2(9Z,12Z)/22:6(4 PE(16:1(9Z)/P-
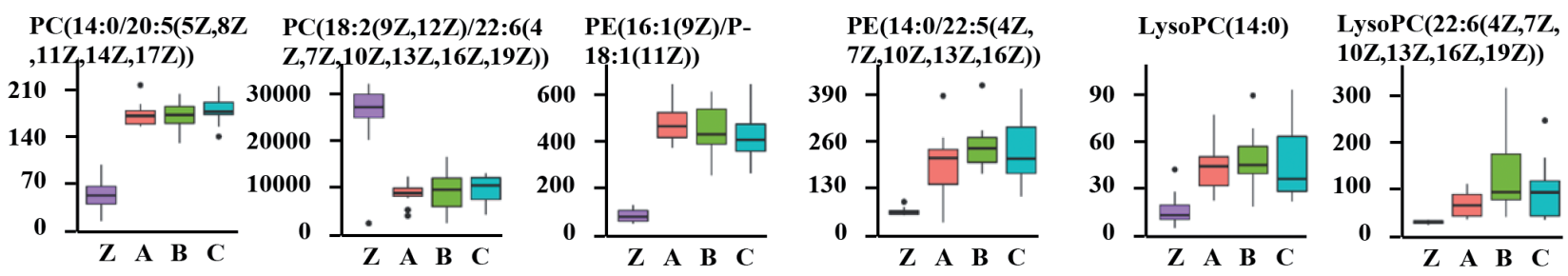

PC(18:4(6Z,9Z,12Z, PC(20:4(5Z,8Z,11Z,14Z)/22: PE(14:0/20:5(5Z,8Z, PE(20:4(5Z,8Z,11Z,14
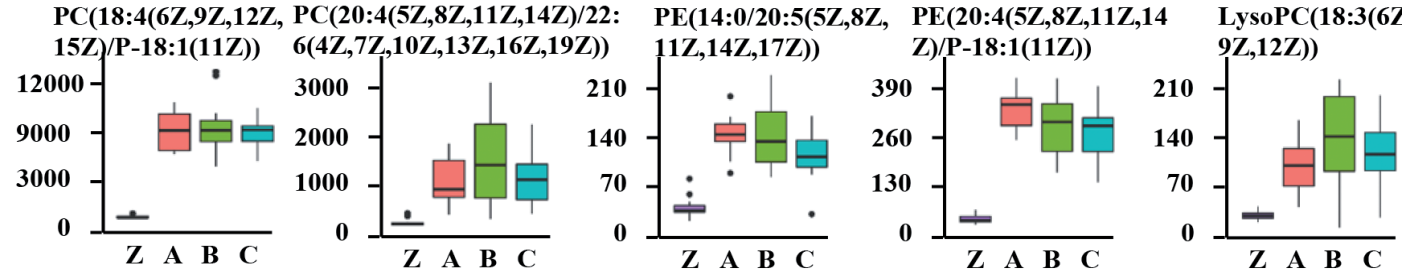

PC(14:0/22:5(4Z,7Z PC(22:4(7Z,10Z,13Z,16Z)/22: PE(18:4(6Z,9Z,12Z,
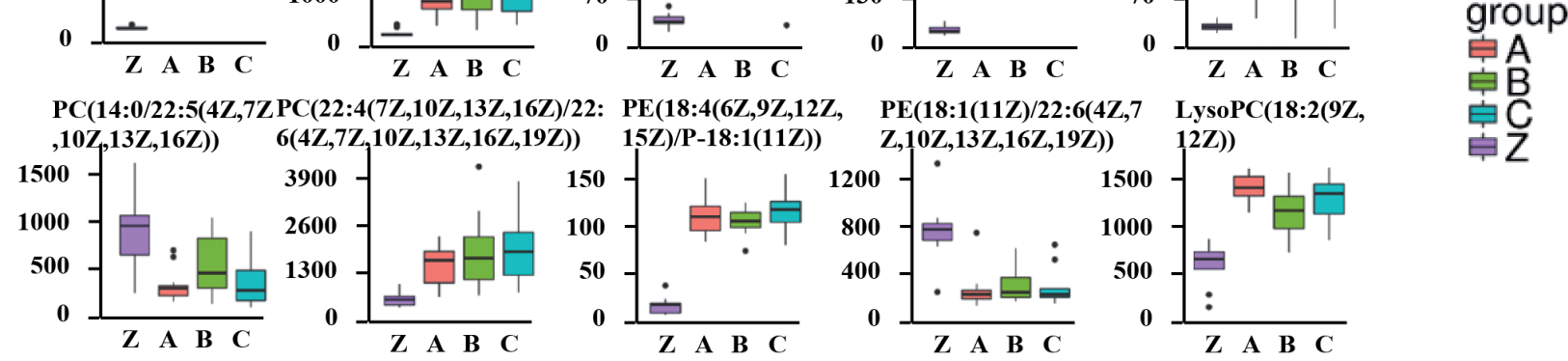

Fig. 4. Relative levels of differential metabolites related to the metabolism of glycerol phosphate esters in liver samples from 4 groups in positive mode. The metabolites, which were involved in glyceryl phosphate metabolism in the liver, including 8 PCs, 9 PEs and 5 LysoPCs were identified and semi-quantitatively measured with LC-QTOF MS. The results revealed that the relative content of phospholipids significantly increased in the TBT-Cl treated group.

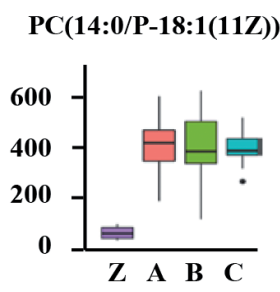

PC(15:0/16:0)

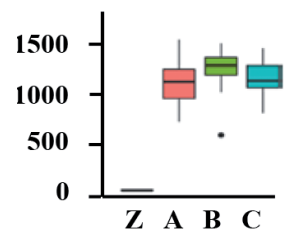

PC(15:0/18:0)

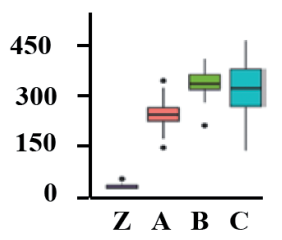

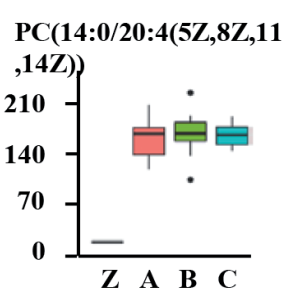

PC(15:0/22:6(4Z,7Z,10Z

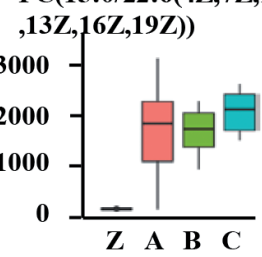

PC(20:0/P-18:1(11Z))

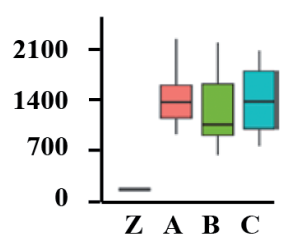

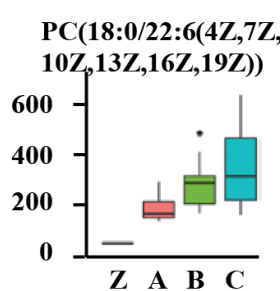
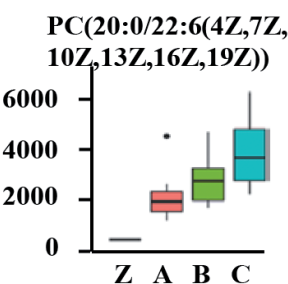

PE(18:3(6Z,9Z,12Z)/20:

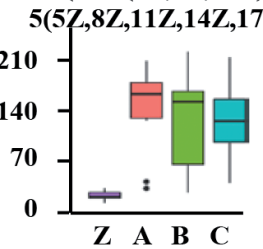

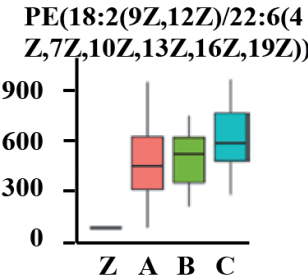

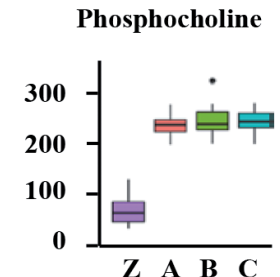

PE(22:5(4Z,7Z,10Z,13Z,16Z)/22 :6(4Z,7Z,10Z,13Z,16Z,19Z))
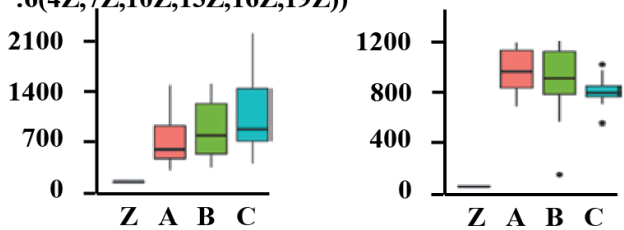

LPA(0:0/16:0)

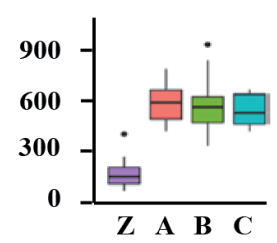

group

泉 $\mathrm{A}$

官B

审乙

Fig. 5. Relative levels of differential metabolites related to the metabolism of glycerol phosphate esters in liver samples from 4 groups in negative mode. The metabolites, which were involved in glyceryl phosphate metabolism in the liver, including 8 PCs, 3 PEs, LPA, phosphocholine and 1 PS were identified and semi-quantitatively measured with LC-QTOF MS. The results revealed that the relative content of phospholipids significantly increased in the TBT-Cl treated group. 


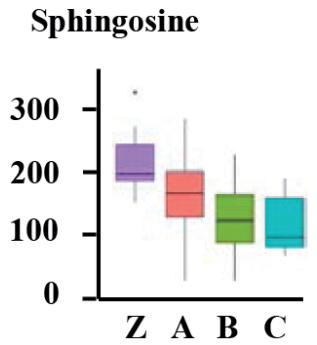

SM(a18:1/12:0)

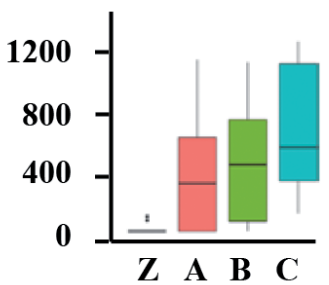

Sphingomyelin

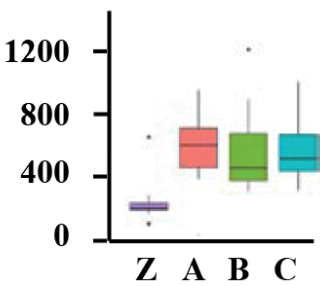

Phytosphingosine

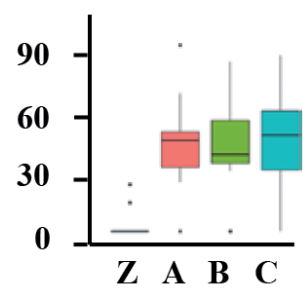

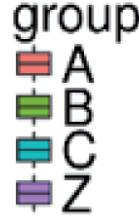

Fig. 6. Relative levels of differential metabolites related to the metabolism of sphingolipids in serum samples from 4 groups in negative mode. The metabolites, which were involved in sphingolipids metabolism in the serum, were identified and semi-quantitatively measured with LC-QTOF MS. The results revealed that the relative content of sphingolipids significantly decreased in the TBT-Cl treated group.

Arachidonic acid

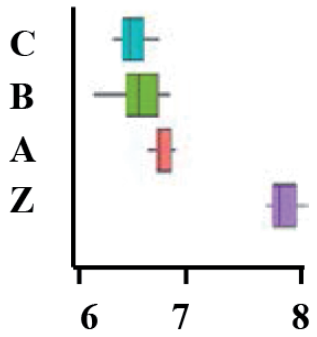

Palmitic acid

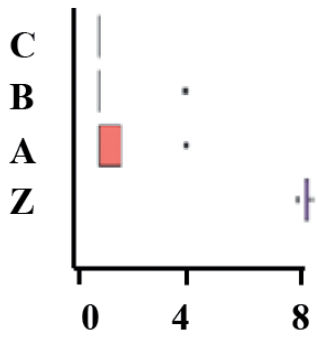

Oleic acid

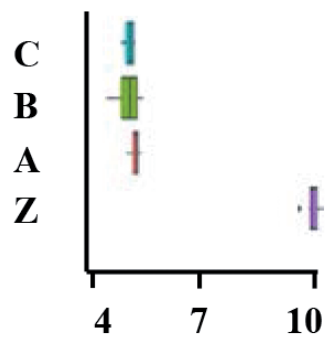

\section{Linoleic acid}

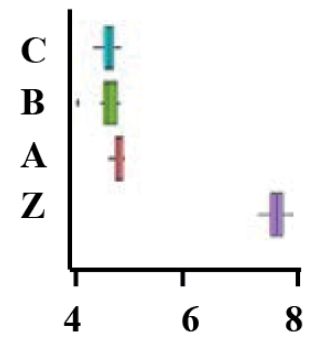

Docosahexaenoic acid

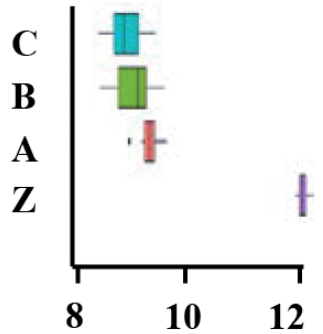

Docosapentaenoic acid

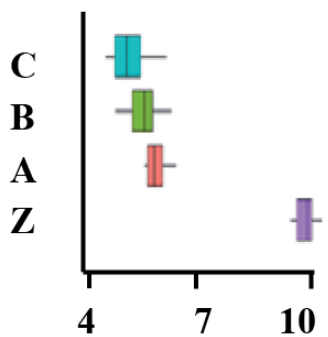

Fig. 7. Relative levels of differential metabolites related to the biosynthesis of unsaturated fatty acids in liver samples from 4 groups in negative mode. The metabolites, involved in unsaturated fatty acids metabolism in the serum, were identified and semi-quantitatively measured with LC-QTOF MS. The results revealed that the relative content of sphingolipids significantly decreased in the TBT-Cl treated group. 
Conversely, the serum sphingosine content in the TBT-Cl treatment groups was significantly lower than that in the control group, and with increasing TBT-Cl concentration, the magnitude of the decline increased, indicating that higher concentrations of TBT-Cl exposure reduced sphingosine synthesis. The decrease of sphingosine in the TBT-Cl treatment groups might be due to the process enhancement of transformation of sphingosine to ceramide, which might negatively affect cell survival, proliferation, cell-cell interactions, and apoptosis.

Histopathological examination showed that the hollow vacuoles of the liver increased significantly in TBT-Cl treatment groups. Moreover, nuclear lysis and even non-nucleated cells were observed, indicating that TBT-Cl exposure caused liver cell death and significant fat accumulation consistent with the effects of TBT-Cl exposure on zebrafish [18]. Arachidonic acid has the potential to intensify stress-reducing responses in fish [19]. In groups A, B, and C, arachidonic acid decreased for $15.93,22.50$, and 16.07 times, respectively, compared with the control group. These results indicated that more arachidonic acid was metabolized in the juvenile T. obscurus under TBT-Cl stimulation (Fig. 7).

\section{Discussion}

In the present study, we used the LC-MS technology to investigate the differential metabolites, including lipids and amino acids, in the liver and serum of juvenile T. obscurus after acute TBT-Cl exposure. The results showed that the differential metabolites in the liver and serum were relatively similar, as were the pathway enrichment results, indicating consistent changes of metabolic and biological process in response to treatments with different concentrations of TBT-Cl. Even at a low concentration (Group A), 96 hours of TBT$\mathrm{Cl}$ exposure had significant effects on the metabolism of juvenile T. obscurus, consistent with the effects of other pollutants on [20]. Therefore, metabolomics could help explain the negative impacts of TBT-Cl exposure on fish metabolisms.

In this research, TBT-Cl exposure directly influenced the metabolism of various essential metabolites in the serum and liver of juvenile T. obscurus, including glyceryl phosphates, sphingolipids, and unsaturated fatty acids. Glyceryl phosphates, including lecithin (PCs), brain phospholipids (PEs), phosphatidylserine (PSs), phosphatidylinositol (PIs), phosphatidylglycerols (PGs), and phosphoglycerides (PAs), are important components of cell membranes and the basis of cell metabolism, energy metabolism, and signal transduction. Generally, juveniles have a weak ability to synthesize phospholipids [21, 22]. In the present study, content of glyceryl phosphates increased in the TBT$\mathrm{Cl}$ treated juvenile T. obscurus and TBT-Cl exposure stimulated phospholipid production. PCs, which are precursors of acetylcholine, are catalyzed by related enzymes to produce choline. However, the content of acetylcholine in juvenile T. obscurus did not increase with the increase of $\mathrm{PC}$ content in this experiment, suggesting that TBT-Cl exposure might disrupt the balance between acetylcholine and acetylcholinesterase [23].

Sphingomyelins, including sphingosine, ceramide, and sphingosine-1-phosphate (S-1-P), are a series of biologically active signaling molecules that are important components of the cell membrane [24]. Sphingomyelin is mainly synthesized by the following two ways: transferring ceramide to the Golgi via ceramide transfer protein, and participating in the production of ceramide. Reportedly, stimulation of exogenous factors like hydrogen peroxide could increase sphingomyelin and ceramide production through remedial synthetic pathways [25]. In this study, TBT$\mathrm{Cl}$ exposure activated and enhanced the sphingomyelin rescue and synthesis pathway in juvenile T. obscurus, leading to the significantly increased sphingomyelin content in serum. Sphingosine, the basic backbone of all sphingolipids, is produced only during deacetylation of ceramide by ceramidase. The metabolomics research results of serum metabolites and liver pathological sections showed that TBT-Cl exposure could reduce sphingosine synthesis, further causing apoptosis.

Previous research indicates that lack of unsaturated

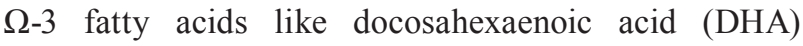
and docosapentaenoic acid (EPA) in food sources caused fat accumulation in fish liver, leading to fatty liver [26]. Generally, appropriate proportions of phospholipids could reduce the risk of fatty liver in fish [27]. However, in response to TBT-Cl exposure, contents of phospholipids increased, leading to a sharp increase in liver fat content. Additionally, in fish, arachidonic acid reportedly increases both resistance to environmental stress and survival rate [19]. Arachidonic acid and its metabolites are of great significance to the lipid metabolism in organisms by regulating lipid accumulation, inhibiting the proliferation, differentiation, and apoptosis of fat cells, promoting the hydrolysis of fat cells, increasing the ability of fatty acid $\beta$-oxidation, and reducing inflammation-related chemokines [28]. It also affects a range of immune functions [29]. However, marine fish generally have a weak ability to synthesize arachidonic acid and therefore need to ingested it from food sources. In this study, in response to TBT-Cl exposure, juvenile T. obscurus consumed more arachidonic acid and produced more $\mathrm{PGE}_{2}$. In addition, lysophospholipids, as raw materials for biofilm synthesis, are involved in the biological processes of most cells [30, 31]. Lysolecithins can affect the immune system by regulating immune cells [32]. In response to TBT-Cl exposure, juvenile T. obscurus experienced an immediate increase of lysolecithin production to improve detoxification function [33], which might reduce the inflammatory responses.

In summary, we used LC-MS technology to detect liver and serum metabolites and identify the differential 
metabolites in juvenile $T$. obscurus after acute exposure to TBT-Cl. The primary differential metabolites were glyceryl phosphate, saturated fatty acids, and amino acids. Further analysis found that the differential metabolites in the serum between the three treatment groups and the control group were mainly related to metabolic pathways including sphingolipid metabolism, phosphoglyceride metabolism, and unsaturated fatty acid biosynthesis. In the liver, the differential metabolites were primarily related to metabolic pathways including phosphoglyceride metabolism, arachidonic acid metabolism, and unsaturated fatty acid biosynthesis. These results provided a basis for understanding the toxicological mechanisms of TBT-Cl to fish.

\section{Conflicts of Interest}

All authors declare no conflict of interest.

\section{Acknowledgements}

This work was supported by the National Infrastructure of Fishery Germplasm Resources (2019DKA3047-003).

\section{References}

1. BARROS A. Tributyltin (TBT) effects at a vascular level: University of Beira Interior. 2016.

2. DE ARAÚJO J.F.P., PODRATZ P.L., SENA G.C., MERLO E., FREITAS-LIMA L.C., AYUB J.G.M., PEREIRA A.F.Z., SANTOS-SILVA A.P., MIRANDA-ALVES L., SILVA I.V., GRACELI J.B. The obesogen tributyltin induces abnormal ovarian adipogenesis in adult female rats. Toxicol. Lett. 295, 99, 2018.

3. PAGLIARANI A., BANDIERA P., VENTRELLA V., TROMBETTI F., PIRINI M., NESCI S., BORGATTI A.R. Tributyltin (TBT) inhibition of oligomycin-sensitive MgATPase activity in mussel mitochondria. Toxicol. In Vitro. 22 (4), 827, 2008.

4. VENTRELLA V., NESCI S., TROMBETTI F., BANDIERA P., PIRINI M., BORGATTI A.R., PAGLIARANI A. Tributyltin inhibits the oligomycinsensitive Mg-ATPase activity in Mytilus galloprovincialis digestive gland mitochondria. Comp. Biochem. Physiol. C Toxicol. Pharmacol. 153 (1), 75, 2011.

5. NESCI S., VENTRELLA V., TROMBETTI F., PIRINI M., BORGATTI A.R., PAGLIARANI A. Tributyltin (TBT) and dibutyltin (DBT) differently inhibit the mitochondrial Mg-ATPase activity in mussel digestive gland. Toxicol. In Vitro. 25 (1), 117, 2011.

6. BURTON J.E., DOROCIAK I.R., SCHWEDLER T.E., RICE C.D. Circulating lysozyme and hepatic CYP1A activities during a chronic dietary exposure to tributyltin (TBT) and 3,3',4,4',5-pentachlorobiphenyl (PCB-126) mixtures in channel catfish, Ictalurus punctatus. J. Toxicol. Enviro. Health A. 65 (8), 589, 2002.

7. KIM S.K., KIM J.H., HAN J.H., YOON Y.D. Inhibitory effect of tributyltin on expression of steroidogenic enzymes in mouse testis. Int. J. Toxicol. 27 (2), 175, 2008.
8. ZHANG C.N., ZHANG J.L., REN H.T., ZHOU B.H., WU Q.J., SUN P. Effect of tributyltin on antioxidant ability and immune responses of zebrafish (Danio rerio). Ecotoxicol. Environ. Saf. 138, 1, 2017.

9. LIANG X., SOUDERS C.L., 2ND, ZHANG J., MARTYNIUK C.J. Tributyltin induces premature hatching and reduces locomotor activity in zebrafish (Danio rerio) embryos/larvae at environmentally relevant levels. Chemosphere. 189, 498, 2017.

10. DAIGNEAULT B.W., LATHAM K.E. 146 Tributyltin chloride exposure alters ejaculated bull sperm function and embryo development. Reprod. Fert. Dev. 32 (2), 199, 2020.

11. GAO J.M. HU J.Y., ZHEN H., YANG M., LI B.Z. Organotin Compounds in the Three Gorges Reservoir Region of the Yangtze River. Bull. Environ. Contam. Toxicol. 76, 155, 2006.

12. CAO D., JIANG G., ZHOU Q., YANG R. Organotin pollution in China: an overview of the current state and potential health risk. J. Environ. Manage. 90, S16, 2009.

13. XU D.P., JIANG S.L., ZHAO C.S., FANG D.A., HU H.Y. Comparative transcriptomics analysis of the river pufferfish (Takifugu obscurus) by tributyltin exposure: Clues for revealing its toxic injury mechanism. Fish Shellfish Immunol. 82, 536, 2018.

14. CHENG C.H., YE C.X., GUO Z.X., WANG A.L. Immune and physiological responses of pufferfish (Takifugu obscurus) under cold stress. Fish Shellfish Immunol. 64, 137, 2017

15. DONG-PO X., DI-AN F., CHANG-SHENG Z., SHU-LUN J., HAO-YUAN H. Effect of tributyltin chloride (TBT-Cl) exposure on expression of HSP90 11 in the river pufferfish (Takifugu obscurus): Evidences for its immunologic function involving in exploring process. Gene. 666, 9, 2018.

16. LI X.Y, ZENG, S.H., DONG X.Y., MA J.G., Wang J.J. Acute toxicity and responses of antioxidant systems to 1-methyl-3-octylimidazolium bromide at different developmental stages of goldfish. Ecotoxicology 21, 253, 2012.

17. IRERHIEVWIE G.O., AKPOGHELIE J.O. Assessment of the Bioaccumulation and the Excretion Rate of $\mathrm{Cd}, \mathrm{Zn}$ and $\mathrm{Pb}$ in Blood, Kidney and Liver of an African Catfish Juvenile in an Artificial Fish Pond. Int. J. Sci. Res. 4, SUB159095, 2015.

18. ZHANG J., SUN P., KONG T., YANG F., GUAN W. Tributyltin promoted hepatic steatosis in zebrafish (Danio rerio) and the molecular pathogenesis involved. Aquat. Toxicol. 170, 208, 2016.

19. BESSONART M., IZQUIERDO M.S., SALHI M., HERNÁNDEZ-CRUZ C.M., GONZÁLEZ M.M., FERNÁNDEZ-PALACIOS $H$. Effect of dietary arachidonic acid levels on growth and survival of gilthead sea bream (Sparus aurata L.) larvae. Aquaculture. 179 (1), 265, 1999.

20. DU Z. Effects of organophosphate flame retardants on zebrafish embryo development and liver metabolism. Nanjing University. 2016 [In Chinese].

21. LI A. Nutrition and Feed Science of Aquatic Animals. China Agricultural Press. 1996 [In Chinese].

22. TOCHER D.R., BENDIKSEN E.A., CAMPBELL P.J., BELL J.G. The role of phospholipids in nutrition and metabolism of teleost fish. Aquaculture. 280 (1), 21, 2008.

23. DENG Q. The influence of organophosphorus pesticides in neural and endocrine function of aquatic animal. 
Genomics and Applied Biology. 36 (11), 4639, 2017 [In Chinese].

24. GAULT C.R., OBEID L.M., HANNUN Y.A. An overview of sphingolipid metabolism: from synthesis to breakdown. Adv. Exp. Med. Biol. 688, 1, 2010.

25. KITATANI K., IDKOWIAK-BALDYS J., HANNUN Y.A. The sphingolipid salvage pathway in ceramide metabolism and signaling. Cell Signal. 20 (6), 1010, 2008.

26. YANG H. Mechanism in tilapia' fatty liver pathological changes of lecithin, choline and inositol in the feed. Guangxi University. 2006 [In Chinese].

27. COUTTEAU P., GEURDEN I., CAMARA M.R., BERGOT P., SORGELOOS P. Review on the dietary effects of phospholipids in fish and crustacean larviculture. Aquaculture. 155 (1), 149, 1997.

28. TIAN J.J. Effects and mechanism analysis of arachidonic acid (ARA) on lipid metabolism of grass carp (Ctenopharygodon idellus). Northwest A \& F University. 2017 [In Chinese].

29. BELL J.G., SARGENT J.R. Arachidonic acid in aquaculture feeds: current status and future opportunities. Aquaculture. 218 (1), 491, 2003.
30. GENDASZEWSKA-DARMACH E. Lysophosphatidic acids, cyclic phosphatidicacids and autotaxin as promising targets in therapies of cancer and other diseases. Acta Biochim. Pol. 55 (2), 227, 2008.

31. YEA K., KIM J., YOON J.H., KWON T., KIM J.H., LEE B.D., LEE H.J., LEE S.J., KIM J.I., LEE T.G., BAEK M.C., PARK H.S., PARK K.S., OHBA M., SUH P.G., RYU S.H. Lysophosphatidylcholine activates adipocyte glucose uptake and lowers blood glucose levels in murine models of diabetes. J. Biol. Chem. 284 (49), 33833, 2009.

32. FAN J. The mechanism and significance of abnormal hemolytic lecithin metabolism in the course of liver cancer. The Second Military Medical University. 2017 [In Chinese].

33. HUANG Y. A study of prostaglandin $\mathrm{E}_{2}$ on alleviating liver indury and enhancing liver regeneration acute liver failure in mice. Fudan University. 2012 [In Chinese].

\section{Supplementary Material}

Table S1. Parameters for PCA models from A-Z, B-Z and C-Z.

\begin{tabular}{|c|c|c|c|c|}
\hline Comparison & Model & Component & Cumulative $\mathrm{R}^{2} \mathrm{X}$ & $\mathrm{Q}^{2}$ \\
\hline $\mathrm{A}(10 \% \mathrm{vsZ})$ & $\mathrm{ESI}^{+}$ & 4 & 0.551 & 0.265 \\
\hline $\mathrm{B}(10 \% \mathrm{vsZ})$ & $\mathrm{ESI}^{-}$ & 5 & 0.653 & 0.191 \\
\hline $\mathrm{C}(20 \% \mathrm{vsZ})$ & $\mathrm{ESI}^{+}$ & 4 & 0.583 & 0.291 \\
\hline $\mathrm{D}(20 \% \mathrm{vsZ})$ & $\mathrm{ESI}^{-}$ & 4 & 0.602 & 0.285 \\
\hline $\mathrm{E}(50 \% \mathrm{vsZ})$ & $\mathrm{ESI}^{+}$ & 5 & 0.643 & 0.28 \\
\hline $\mathrm{F}(50 \% \mathrm{vsZ})$ & $\mathrm{ESI}^{-}$ & 4 & 0.6 & 0.307 \\
\hline
\end{tabular}

Table S2. Parameters for OPLS-DA models from A-Z, B-Z and C-Z.

\begin{tabular}{|c|c|c|c|c|c|}
\hline Comparison & Model & Component & $\mathrm{R}^{2} \mathrm{X}$ & $\mathrm{R}^{2} \mathrm{Y}$ & $\mathrm{Q}^{2}$ \\
\hline $\mathrm{A}(10 \% \mathrm{vsZ})$ & $\mathrm{ESI}^{+}$ & $1+1$ & 0.306 & 0.976 & 0.918 \\
\hline $\mathrm{B}(10 \% \mathrm{vsZ})$ & $\mathrm{ESI}^{-}$ & $2+1$ & 0.443 & 0.995 & 0.948 \\
\hline $\mathrm{C}(20 \% \mathrm{vsZ})$ & $\mathrm{ESI}^{+}$ & $2+1$ & 0.397 & 0.988 & 0.91 \\
\hline $\mathrm{D}(20 \% \mathrm{vsZ})$ & $\mathrm{ESI}^{-}$ & $2+1$ & 0.474 & 0.984 & 0.928 \\
\hline $\mathrm{E}(50 \% \mathrm{vsZ})$ & $\mathrm{ESI}^{+}$ & $2+1$ & 0.464 & 0.985 & 0.922 \\
\hline $\mathrm{F}(50 \% \mathrm{vsZ})$ & $\mathrm{ESI}^{-}$ & $3+1$ & 0.476 & 0.998 & 0.924 \\
\hline
\end{tabular}


Table S3. Parameters for PCA models from A-Z, B-Z and C-Z.

\begin{tabular}{|l|c|c|c|c|}
\hline Comparison & Model & Component & $\mathrm{R}^{2} \mathrm{X}$ & $\mathrm{R}^{2} \mathrm{Y}$ \\
\hline $\mathrm{A}(10 \% \mathrm{vsZ})$ & $\mathrm{ESI}^{+}$ & 3 & 0.637 & 0.461 \\
\hline $\mathrm{B}(10 \% \mathrm{vsZ})$ & $\mathrm{ESI}^{-}$ & 2 & 0.703 & 0.623 \\
\hline $\mathrm{C}(20 \% \mathrm{vsZ})$ & $\mathrm{ESI}^{+}$ & 3 & 0.649 & 0.478 \\
\hline $\mathrm{D}(20 \% \mathrm{vsZ})$ & $\mathrm{ESI}^{-}$ & 3 & 0.745 & 0.643 \\
\hline $\mathrm{E}(50 \% \mathrm{vsZ})$ & $\mathrm{ESI}^{+}$ & 2 & 0.583 & 0.473 \\
\hline $\mathrm{F}(50 \% \mathrm{vsZ})$ & $\mathrm{ESI}^{-}$ & 2 & 0.696 & 0.604 \\
\hline
\end{tabular}

Table S4. Parameters for OPLS-DA models from A-Z,B-Z and C-Z.

\begin{tabular}{|c|c|c|c|c|c|}
\hline Comparison & Model & Component & $\mathrm{R}^{2} \mathrm{X}$ & $\mathrm{R}^{2} \mathrm{Y}$ & $\mathrm{Q}^{2}$ \\
\hline $\mathrm{A}(10 \% \mathrm{vsZ})$ & $\mathrm{ESI}^{+}$ & $1+1$ & 0.539 & 0.999 & 0.995 \\
\hline $\mathrm{B}(10 \% \mathrm{vsZ})$ & $\mathrm{ESI}^{-}$ & $1+1$ & 0.674 & 1 & 0.998 \\
\hline $\mathrm{C}(20 \% \mathrm{vsZ})$ & $\mathrm{ESI}^{+}$ & $1+1$ & 0.565 & 0.997 & 0.991 \\
\hline $\mathrm{D}(20 \% \mathrm{vsZ})$ & $\mathrm{ESI}^{-}$ & $1+1$ & 0.693 & 0.999 & 0.998 \\
\hline $\mathrm{E}(50 \% \mathrm{vsZ})$ & $\mathrm{ESI}^{+}$ & $1+1$ & 0.556 & 0.999 & 0.993 \\
\hline $\mathrm{F}(50 \% \mathrm{vsZ})$ & $\mathrm{ESI}^{-}$ & $1+1$ & 0.688 & 0.999 & 0.997 \\
\hline
\end{tabular}

Table S5. The differential metabolites in serum between the treatment groups and the control group in positive model.

\begin{tabular}{|c|c|c|c|c|c|}
\hline \multirow{2}{*}{ Differential metabolites } & \multirow{2}{*}{$\begin{array}{l}\text { Molecular } \\
\text { Weight }\end{array}$} & \multirow{2}{*}{$\begin{array}{c}\mathrm{RT} \\
(\mathrm{min})\end{array}$} & \multicolumn{3}{|c|}{$\log _{2}($ FoldChange $)$} \\
\hline & & & A VS Z & B VS Z & C VS Z \\
\hline 1-O-(cis-9-Octadecenyl)-2-O-acetyl-sn-glycero-3-phosphocholine & 549.38 & 11.96 & & & -0.92 \\
\hline 2-(3'-Methylthio)propylmalic acid & 222.06 & 13.19 & 24.10 & 22.49 & 23.28 \\
\hline 2,5-Furandicarboxylic acid & 156.01 & 1.06 & -0.66 & -0.58 & -1.30 \\
\hline 2-aminohexadecanoic acid & 271.25 & 8.74 & 4.76 & 4.81 & 4.91 \\
\hline 2-Hydroxycinnamic acid & 164.05 & 0.77 & & -1.68 & \\
\hline 2-Phenylethyl hexanoate & 220.15 & 11.11 & -1.15 & & \\
\hline 3-Methylxanthine & 166.05 & 5.94 & 25.12 & 24.16 & 24.93 \\
\hline 3-Phosphoglycerol-glutathione & 461.07 & 0.69 & -1.47 & & \\
\hline 4-aminohippurate & 222.09 & 11.96 & 23.44 & 23.46 & 23.48 \\
\hline 4-Aminosalicylic acid & 153.03 & 4.01 & -1.15 & -1.26 & -1.07 \\
\hline 4-Heptyloxyphenol & 208.15 & 11.30 & 23.83 & 24.05 & 24.02 \\
\hline 5'-Guanylylmethylenebisphosphonate & 521.01 & 0.68 & -2.00 & & \\
\hline 6-Methylthioguanine & 181.04 & 0.71 & -0.85 & -2.21 & -1.71 \\
\hline 7-ketodeoxycholic acid & 406.29 & 9.62 & 3.78 & 4.34 & 4.10 \\
\hline Acetylcholine & 145.11 & 0.69 & -1.50 & -1.20 & -1.39 \\
\hline Aleprylic acid & 196.15 & 10.77 & 4.39 & 4.46 & 4.62 \\
\hline Argininosuccinic acid & 290.11 & 8.56 & 28.02 & 27.97 & 27.96 \\
\hline Capric acid & 172.15 & 8.50 & 3.40 & 3.51 & 3.53 \\
\hline Citramalic acid & 148.04 & 5.92 & 2.98 & 1.98 & 2.80 \\
\hline $\mathrm{CPA}(18: 1(11 Z) / 0: 0)$ & 418.26 & 11.98 & 22.34 & 22.92 & 22.70 \\
\hline
\end{tabular}


Table S5. Continued.

\begin{tabular}{|c|c|c|c|c|c|}
\hline Dehydroascorbic acid & 174.02 & 0.78 & -1.10 & -1.16 & -1.01 \\
\hline Dehydrojuvabione & 264.17 & 11.44 & 2.82 & 3.02 & 3.10 \\
\hline Desmosine & 525.29 & 10.08 & -0.65 & -1.61 & -2.23 \\
\hline D-myo-Inositol-2,3,4,5-tetraphosphate & 501.29 & 10.11 & & -2.10 & \\
\hline dodecanamide & 199.19 & 10.60 & 2.24 & 2.49 & 2.37 \\
\hline Dodecanoylcarnitine & 343.27 & 8.59 & -0.83 & -0.66 & \\
\hline Dodecylphosphocholine & 351.24 & 12.47 & 23.98 & 23.61 & 23.79 \\
\hline Eicosatrienoic acid & 306.24 & 9.29 & 1.28 & 1.31 & 1.18 \\
\hline Fenamic acid & 213.08 & 8.86 & -1.46 & -1.52 & -1.35 \\
\hline Glucoheptonic acid & 226.07 & 4.08 & -1.69 & -2.10 & -1.64 \\
\hline Guanosine & 283.09 & 1.42 & & & -1.35 \\
\hline Hexadecanedioic acid & 286.21 & 12.86 & 6.08 & 5.29 & 5.68 \\
\hline Homocarnosine & 240.14 & 9.75 & -1.18 & -0.87 & -0.83 \\
\hline L-Arginine & 254.08 & 5.66 & -1.63 & -1.51 & -1.42 \\
\hline L-Arginine phosphate & 174.11 & 0.67 & -1.29 & -2.86 & -1.87 \\
\hline L-Carnitine & 161.11 & 0.68 & -1.67 & & \\
\hline L-Formylkynurenine & 236.07 & 7.93 & 2.60 & 2.48 & 2.40 \\
\hline L-Histidine & 155.07 & 0.64 & & -1.88 & -1.81 \\
\hline Linderic acid & 198.16 & 9.84 & -2.69 & -2.58 & -2.71 \\
\hline Linoleyl carnitine & 423.33 & 9.93 & -1.05 & -1.09 & -0.95 \\
\hline Lithocholic acid & 376.32 & 13.28 & & & 1.12 \\
\hline $\operatorname{LPA}(0: 0 / 16: 0)$ & 410.23 & 4.03 & 3.22 & & 2.90 \\
\hline L-Phenylalanine & 165.08 & 2.19 & 0.85 & & \\
\hline L-Tryptophan & 204.08 & 11.99 & 6.10 & 6.17 & 6.20 \\
\hline LysoPC(18:1(11Z)) & 521.35 & 10.98 & & & 1.11 \\
\hline LysoPC(20:4(5Z,8Z,11Z,14Z)) & 543.33 & 10.18 & & & -1.30 \\
\hline LysoPC(22:5(4Z,7Z,10Z,13Z,16Z)) & 569.35 & 10.36 & & & -1.37 \\
\hline LysoPC(22:6(4Z,7Z,10Z,13Z,16Z,19Z)) & 567.33 & 10.17 & & -1.41 & -1.74 \\
\hline LysoPC(P-16:0) & 479.34 & 10.41 & 1.51 & & \\
\hline LysoPE(0:0/16:0) & 453.29 & 10.57 & & -0.89 & \\
\hline LysoPE(0:0/18:1(11Z)) & 479.30 & 10.90 & & -0.99 & \\
\hline LysoPE(0:0/18:2(9Z,12Z)) & 477.29 & 10.15 & & & -0.81 \\
\hline LysoPE(0:0/22:1(13Z)) & 535.36 & 11.52 & & & -1.50 \\
\hline Malic acid & 134.02 & 1.42 & & & -1.16 \\
\hline MG(0:0/22:6(4Z,7Z,10Z,13Z,16Z,19Z)/0:0) & 402.28 & 12.65 & & 2.35 & 2.50 \\
\hline Oleamide & 281.27 & 9.20 & & -1.10 & -2.12 \\
\hline O-Phospho-DL-Tyrosine & 261.03 & 0.83 & & & -1.26 \\
\hline Oxoglutaric acid & 146.02 & 0.78 & -0.76 & -0.66 & -0.60 \\
\hline $\operatorname{PA}(6: 0 / 6: 0)$ & 368.16 & 9.03 & 23.24 & 23.71 & 23.39 \\
\hline Palmitic amide & 255.26 & 13.43 & & & 1.84 \\
\hline
\end{tabular}


Table S5. Continued.

\begin{tabular}{|c|c|c|c|c|c|}
\hline Palmitoyl-L-carnitine & 399.33 & 10.25 & -0.96 & & \\
\hline $\mathrm{PC}(16: 0 / 5: 0(\mathrm{COOH}))$ & 609.36 & 11.02 & 1.28 & & \\
\hline $\mathrm{PC}(\mathrm{O}-20: 0 / 0: 0)$ & 537.42 & 14.05 & 1.76 & 2.38 & 2.35 \\
\hline $\mathrm{PE}(\mathrm{P}-16: 0 / 0: 0)$ & 437.29 & 10.94 & -0.63 & & -0.71 \\
\hline Phytosphingosine & 317.29 & 8.97 & 3.94 & 3.76 & 3.83 \\
\hline $\mathrm{PS}(12: 0 / 12: 0)$ & 623.38 & 12.22 & 2.21 & & 1.39 \\
\hline $\operatorname{PS}(12: 0 / 14: 1(9 Z))$ & 649.40 & 13.08 & 2.89 & & 2.30 \\
\hline PS(13:0/18:3(6Z,9Z,12Z)) & 715.44 & 12.16 & 4.77 & 2.81 & 3.96 \\
\hline $\operatorname{PS}(21: 0 / 0: 0)$ & 567.35 & 10.88 & 1.57 & & \\
\hline $\operatorname{PS}(22: 1(11 Z) / 0: 0)$ & 579.35 & 11.31 & 1.95 & & \\
\hline $\operatorname{PS}(22: 2(13 Z, 16 Z) / 0: 0)$ & 577.41 & 13.56 & & & 0.97 \\
\hline psychosine sulfate & 541.32 & 9.67 & -1.39 & & \\
\hline Riboflavin (Vitamin B2) & 376.14 & 4.87 & -1.48 & -2.10 & -1.66 \\
\hline Scopoletin & 192.04 & 5.66 & -1.57 & -1.51 & 5.12 \\
\hline $\operatorname{SM}(\mathrm{d} 18: 1 / 12: 0)$ & 646.50 & 14.68 & 4.91 & 4.95 & 5.59 \\
\hline Sphinganine & 301.30 & 8.92 & 1.43 & 1.28 & 1.31 \\
\hline Sphingosine & 299.28 & 9.19 & & -0.92 & -1.05 \\
\hline Squalamine & 627.49 & 13.86 & 1.62 & & -1.04 \\
\hline Tetraacetylethylenediamine & 228.11 & 0.72 & & -1.61 & -1.76 \\
\hline Tetradecanoylcarnitine & 371.30 & 9.42 & -0.74 & & \\
\hline Tranexamic acid & 157.11 & 0.99 & 1.08 & & 0.93 \\
\hline trans-trans-muconic acid & 142.02 & 0.66 & 3.97 & 4.46 & 3.78 \\
\hline UDP-D-galacturonate & 580.01 & 0.98 & & & 1.04 \\
\hline Vaccenyl carnitine & 425.35 & 10.46 & -1.18 & -1.48 & \\
\hline Vanilpyruvic acid & 210.05 & 5.41 & -1.51 & -1.62 & -1.07 \\
\hline$\beta$-Hydroxyisovaleric acid & 118.07 & 4.01 & -0.97 & -1.01 & -0.86 \\
\hline
\end{tabular}


Table S6. The differential metabolites in serum between the treatment groups and the control group in negative model.

\begin{tabular}{|c|c|c|c|c|c|}
\hline \multirow{2}{*}{ Differential metabolites } & \multirow{2}{*}{$\begin{array}{c}\text { Molecular } \\
\text { Weight }\end{array}$} & \multirow{2}{*}{$\begin{array}{l}\text { RT } \\
(\min )\end{array}$} & \multicolumn{3}{|c|}{$\log _{2}($ FoldChange $)$} \\
\hline & & & A VS Z & B VS Z & C VS Z \\
\hline 3-Formylsalicylic acid & 166.03 & 5.01 & 1.32 & & \\
\hline 3-Methylxanthine & 166.05 & 0.68 & & 3.05 & 3.00 \\
\hline 5'-Guanylylmethylenebisphosphonate & 521.01 & 0.66 & -1.76 & & \\
\hline 5Z,11Z,14Z,17Z-eicosatetraenoic acid & 304.24 & 13.58 & -2.16 & -2.59 & -2.33 \\
\hline ADP-ribose & 559.07 & 0.98 & & 1.74 & \\
\hline Aleprylic acid & 196.15 & 9.78 & 0.51 & 0.72 & 0.70 \\
\hline AV-Ceramide & 655.31 & 9.65 & 2.48 & & 2.08 \\
\hline Benfuresate & 256.24 & 14.37 & -1.98 & -2.24 & -1.71 \\
\hline Cholic acid glucuronide & 584.34 & 10.87 & -3.08 & -2.62 & -2.96 \\
\hline Clupanodonic acid & 330.26 & 13.71 & -3.72 & -3.78 & -3.28 \\
\hline Dehydrochorismic acid & 224.04 & 8.63 & & -1.20 & \\
\hline Desmosine & 525.29 & 10.15 & & -1.11 & \\
\hline D-myo-Inositol-2,3,4,5-tetraphosphate & 499.92 & 0.58 & & 0.94 & \\
\hline Eicosapentaenoic Acid & 302.22 & 12.97 & & & -1.30 \\
\hline Homocarnosine & 240.14 & 9.78 & & 0.48 & 0.38 \\
\hline L-Homotyrosine & 195.09 & 10.02 & 1.02 & 1.48 & 1.43 \\
\hline Linoleic acid & 280.24 & 13.74 & -2.91 & -3.24 & -2.61 \\
\hline L-Phenylalanine & 165.08 & 2.18 & 1.44 & 1.03 & 1.44 \\
\hline L-Tyrosine & 181.07 & 1.16 & -1.32 & -1.44 & \\
\hline LysoPE(0:0/20:1(11Z)) & 507.33 & 10.93 & & -1.00 & -1.04 \\
\hline LysoPE(0:0/22:5(4Z,7Z,10Z,13Z,16Z)) & 527.30 & 10.40 & -1.11 & -1.88 & -1.44 \\
\hline LysoPE(0:0/24:6(6Z,9Z,12Z,15Z,18Z,21Z)) & 553.32 & 10.23 & -0.72 & & -0.77 \\
\hline Mesaconic acid & 130.03 & 0.84 & & & -1.68 \\
\hline Ne-Methyl-L-lysine & 160.12 & 0.71 & & 0.64 & 0.45 \\
\hline Nonanedioic acid & 188.10 & 6.29 & -1.65 & -1.32 & -1.39 \\
\hline N $\alpha$-Acetyl-L-glutamine & 188.08 & 0.83 & 1.17 & 1.78 & 1.23 \\
\hline Oleic Acid & 282.26 & 14.54 & -2.85 & -3.24 & -2.69 \\
\hline $\mathrm{PC}(16: 0 / 5: 0(\mathrm{CHO}))$ & 593.37 & 11.31 & -0.78 & & \\
\hline $\operatorname{PS}(12: 0 / 12: 0)$ & 623.38 & 12.26 & 1.33 & & \\
\hline $\operatorname{PS}(18: 2(9 Z, 12 Z) / 0: 0)$ & 521.27 & 10.61 & 0.72 & 1.06 & \\
\hline Pseudoaconitine & 689.35 & 11.85 & 1.65 & & \\
\hline Taurocholic acid & 515.29 & 7.69 & & 23.93 & \\
\hline Vanilpyruvic acid & 210.05 & 5.45 & & 1.46 & 1.16 \\
\hline
\end{tabular}


Table S7. The differential metabolites in liver between the treatment groups and the control group in positive model.

\begin{tabular}{|c|c|c|c|c|c|}
\hline \multirow{2}{*}{ Differential metabolites } & \multirow{2}{*}{$\begin{array}{l}\text { Molecular } \\
\text { Weight }\end{array}$} & \multirow{2}{*}{$\begin{array}{l}\mathrm{RT} \\
(\mathrm{min})\end{array}$} & \multicolumn{3}{|c|}{$\log _{2}($ FoldChange $)$} \\
\hline & & & A vS Z & B VS Z & C vs Z \\
\hline 11-Hydroxyandrosterone & 306.22 & 10.02 & & & 0.75 \\
\hline 19,20-DiHDPA & 362.25 & 12.67 & 3.14 & 3.31 & 3.35 \\
\hline 2,3-dihydrobenzofuran & 120.06 & 2.31 & -0.86 & -1.03 & -1.03 \\
\hline 2-Phenylethanol glucuronide & 298.11 & 4.28 & 5.55 & 5.53 & 5.98 \\
\hline 3-Hydroxyanthranilic acid & 153.04 & 1.16 & -2.05 & -1.34 & -1.82 \\
\hline 3-keto-2-methylbutyrate & 115.04 & 11.82 & 3.39 & 3.24 & 3.23 \\
\hline 3-Oxo-4,6-choladienoic acid & 370.25 & 9.19 & & 1.50 & \\
\hline 4a-Hydroxytetrahydrobiopterin & 257.11 & 1.63 & 2.48 & 2.15 & 2.40 \\
\hline 4-Guanidinobutanoic acid & 145.09 & 0.77 & -1.45 & -1.32 & -1.16 \\
\hline 4-Hydroxyphenylacetylglutamine & 281.09 & 0.69 & -2.02 & -2.17 & -2.01 \\
\hline 5,8-Tetradecadienoic acid & 224.18 & 6.51 & 0.70 & 0.82 & 0.92 \\
\hline 5-Hydroxykynurenamine & 180.09 & 1.72 & 1.59 & 1.88 & 1.47 \\
\hline 5-Methoxydimethyltryptamine & 218.14 & 1.44 & & 1.53 & 1.47 \\
\hline 6-Methyladenine & 149.07 & 0.78 & -2.24 & -2.44 & -2.90 \\
\hline 7,8-Dihydroneopterin & 255.10 & 5.93 & -1.19 & -1.18 & -1.04 \\
\hline 7-Sulfocholic acid & 488.24 & 1.66 & & 1.15 & \\
\hline 8-Hydroxypurine & 138.05 & 1.08 & -1.86 & -1.47 & -1.45 \\
\hline Adenosine & 267.10 & 2.94 & 7.79 & 7.80 & 7.79 \\
\hline ADP-Ribosyl-L-arginine & 715.17 & 10.57 & 7.64 & 7.69 & 7.67 \\
\hline Arachidonic Acid & 304.24 & 13.29 & -3.99 & -4.49 & -4.01 \\
\hline Argininosuccinic acid & 290.12 & 8.86 & & 4.23 & 5.81 \\
\hline Bufotenine & 204.13 & 5.26 & 2.68 & 2.74 & 2.67 \\
\hline Cholic acid & 408.29 & 11.46 & -1.56 & -1.23 & -1.32 \\
\hline Chorismic acid & 226.05 & 5.79 & 2.79 & 2.41 & 2.94 \\
\hline Citrulline & 175.10 & 0.70 & -2.81 & -2.62 & -3.24 \\
\hline Corticosterone & 346.21 & 12.65 & 4.38 & 4.10 & 4.37 \\
\hline Cysteine-S-sulfate & 200.98 & 0.55 & 3.51 & 3.29 & 3.39 \\
\hline dCMP & 307.06 & 1.57 & 2.93 & 3.15 & 3.03 \\
\hline Deoxyadenosine & 251.10 & 2.69 & 3.42 & 3.52 & 3.55 \\
\hline Deoxyinosine & 252.09 & 1.40 & 2.28 & 2.27 & 2.18 \\
\hline Deoxyuridine monophosphate (dUMP) & 308.04 & 1.58 & 3.73 & 3.92 & 3.75 \\
\hline DG(15:0/20:5(5Z,8Z,11Z,14Z,17Z)/0:0) & 600.48 & 12.70 & 0.95 & 1.00 & 1.00 \\
\hline DG(15:0/22:5(4Z,7Z,10Z,13Z,16Z)/0:0) & 628.51 & 14.28 & & 0.63 & \\
\hline DG(20:2(11Z,14Z)/22:5(4Z,7Z,10Z,13Z,16Z)/0:0) & 694.55 & 11.77 & 2.72 & 2.87 & 2.82 \\
\hline DG(20:4(8Z,11Z,14Z,17Z)/22:6(4Z,7Z,10Z,13Z,16Z,19Z)/0:0) & 688.51 & 12.57 & -1.05 & & \\
\hline Diethylphosphate & 154.04 & 4.20 & -3.27 & -3.18 & -3.07 \\
\hline Dihydrothymine & 128.06 & 1.58 & -2.38 & -2.51 & -2.39 \\
\hline Dodecanedioic acid & 230.15 & 1.35 & & 2.36 & 2.84 \\
\hline
\end{tabular}


Table S7. Continued.

\begin{tabular}{|c|c|c|c|c|c|}
\hline Elaidic carnitine & 425.35 & 10.13 & & 2.21 & \\
\hline Homoarginine & & & 2.89 & 2.84 & 2.81 \\
\hline Homogentisic acid & & & 3.27 & 2.81 & 3.46 \\
\hline Ketoleucine & & & -1.82 & -1.74 & -1.99 \\
\hline Leukotriene E3 & 441.25 & 11.10 & 3.33 & & \\
\hline L-Hydroxylysine & 162.10 & 0.76 & -1.51 & -1.00 & \\
\hline LysoPC(14:0) & 467.30 & 11.49 & 1.68 & 1.73 & \\
\hline LysoPC(18:2(9Z,12Z)) & 519.33 & 10.89 & 0.94 & 0.66 & 0.85 \\
\hline LysoPC(18:3(6Z,9Z,12Z)) & 517.32 & 9.42 & 1.84 & 2.23 & 2.03 \\
\hline LysoPC $(20: 3(5 Z, 8 Z, 11 Z))$ & 545.35 & 11.17 & 1.25 & & 1.20 \\
\hline LysoPC(22:6(4Z,7Z,10Z,13Z,16Z,19Z)) & 567.33 & 9.90 & 2.26 & & \\
\hline $\operatorname{MG}(0: 0 / 18: 3(6 Z, 9 Z, 12 Z) / 0: 0)$ & 352.26 & 11.93 & 1.68 & 1.37 & 1.59 \\
\hline $\mathrm{MG}(0: 0 / 20: 2(11 \mathrm{Z}, 14 \mathrm{Z}) / 0: 0)$ & 382.31 & 9.49 & 1.98 & 1.95 & 2.18 \\
\hline $\mathrm{MG}(0: 0 / 20: 3(11 Z, 14 Z, 17 Z) / 0: 0)$ & 380.29 & 13.11 & -2.20 & -2.16 & -2.11 \\
\hline $\mathrm{MG}(0: 0 / 20: 4(8 Z, 11 Z, 14 Z, 17 Z) / 0: 0)$ & 378.28 & 12.50 & 1.94 & 1.67 & 1.84 \\
\hline $\operatorname{MG}(0: 0 / 20: 5(5 Z, 8 Z, 11 Z, 14 Z, 17 Z) / 0: 0)$ & 376.26 & 9.97 & 0.73 & 0.74 & 0.88 \\
\hline N-Acetyl-L-aspartic acid & 175.05 & 1.22 & 1.16 & 1.32 & 1.15 \\
\hline N-Acetyl-L-tyrosine & 223.08 & 8.90 & -2.64 & -2.83 & -2.84 \\
\hline Nicotinamide riboside & 254.09 & 0.78 & & & -0.61 \\
\hline N-Methyl-4-pyridone-3-carboxamide & 152.06 & 1.80 & 3.93 & 3.79 & 3.94 \\
\hline n-Ribosylhistidine & 287.11 & 8.87 & & 2.55 & 4.21 \\
\hline Palmitic amide & 255.26 & 8.54 & 2.99 & 2.97 & 2.90 \\
\hline p-Aminobenzoic acid & 137.05 & 1.08 & -1.31 & -1.29 & -1.22 \\
\hline $\mathrm{PC}(14: 0 / 20: 5(5 Z, 8 Z, 11 Z, 14 Z, 17 Z))$ & 751.52 & 13.23 & 1.52 & 1.49 & 1.58 \\
\hline $\mathrm{PC}(14: 0 / 22: 5(4 \mathrm{Z}, 7 \mathrm{Z}, 10 \mathrm{Z}, 13 \mathrm{Z}, 16 \mathrm{Z}))$ & 779.55 & 14.14 & -1.73 & & -1.63 \\
\hline PC(15:0/16:1(9Z)) & 717.53 & 12.90 & -1.82 & -1.35 & -1.43 \\
\hline PC(16:1(9Z)/22:6(4Z,7Z,10Z,13Z,16Z,19Z)) & 803.55 & 14.85 & 5.70 & & \\
\hline PC(18:2(9Z,12Z)/22:6(4Z,7Z,10Z,13Z,16Z,19Z)) & 829.56 & 13.19 & -1.55 & -1.41 & -1.40 \\
\hline PC(18:4(6Z,9Z,12Z,15Z)/P-18:1(11Z)) & 763.55 & 13.17 & 3.88 & 3.89 & 3.82 \\
\hline PC(20:4(5Z,8Z,11Z,14Z)/22:6(4Z,7Z,10Z,13Z,16Z,19Z)) & 853.56 & 14.76 & 3.74 & 4.25 & 3.86 \\
\hline PC(22:4(7Z,10Z,13Z,16Z)/22:6(4Z,7Z,10Z,13Z,16Z,19Z)) & 881.59 & 14.55 & 2.46 & & 2.92 \\
\hline $\operatorname{PE}(14: 0 / 20: 5(5 Z, 8 Z, 11 Z, 14 Z, 17 Z))$ & 709.47 & 14.32 & 1.80 & 1.77 & 1.48 \\
\hline $\operatorname{PE}(14: 0 / 22: 5(4 Z, 7 Z, 10 Z, 13 Z, 16 Z))$ & 737.50 & 14.77 & 2.41 & 2.76 & 2.69 \\
\hline $\operatorname{PE}(16: 1(9 Z) / P-18: 1(11 Z))$ & 699.52 & 14.08 & 2.75 & 2.61 & 2.54 \\
\hline PE(18:1(11Z)/22:6(4Z,7Z,10Z,13Z,16Z,19Z)) & 789.53 & 12.94 & -1.96 & -1.63 & -1.83 \\
\hline PE(18:1(11Z)/P-18:1(11Z)) & 727.55 & 14.81 & 1.16 & 0.98 & \\
\hline PE(18:3(6Z,9Z,12Z)/P-16:0) & 697.50 & 14.08 & 7.05 & 6.96 & 6.84 \\
\hline PE(18:4(6Z,9Z,12Z,15Z)/P-18:1(11Z)) & 721.50 & 14.06 & 2.70 & 2.58 & 2.77 \\
\hline PE(20:4(5Z,8Z,11Z,14Z)/P-18:1(11Z)) & 749.54 & 14.84 & 3.04 & 2.82 & 2.76 \\
\hline PE(22:4(7Z,10Z,13Z,16Z)/22:5(4Z,7Z,10Z,13Z,16Z)) & 841.56 & 14.86 & 4.89 & 5.14 & 4.94 \\
\hline
\end{tabular}


Table S7. Continued.

\begin{tabular}{|c|c|c|c|c|c|}
\hline PGE2 & 352.23 & 9.69 & 2.74 & 2.97 \\
\hline Phenylglyoxylic acid & 150.03 & 12.36 & -1.18 & -1.33 & -1.70 \\
\hline Phosphatidylserine & 385.11 & 3.97 & 4.86 & 4.68 & 4.46 \\
\hline Pseudomonine & 330.13 & 8.86 & & 3.54 & 5.21 \\
\hline psychosine sulfate & 541.29 & 9.38 & 1.57 & 1.49 & 1.47 \\
\hline S-Adenosylhomocysteine & 384.12 & 3.97 & 6.88 & 6.74 & 6.47 \\
\hline S-Adenosylmethioninamine & 354.15 & 1.69 & & 2.06 & \\
\hline Spermine & 202.22 & 7.38 & 2.65 & 2.72 & 2.96 \\
\hline Squalene & 410.39 & 8.45 & 2.14 & 2.00 & -2.01 \\
\hline Tetradecanedioic acid & 258.18 & 12.73 & & 3.22 & \\
\hline Tocopheronic acid & 294.15 & 8.89 & -3.40 & -3.61 & 2.18 \\
\hline trifluoroacetic acid & 113.99 & 0.58 & -1.43 & & \\
\hline Trimethylammonioacetate & 118.09 & 0.84 & 0.78 & \\
\hline
\end{tabular}

Table S8. The differential metabolites in liver between the treatment groups and the control group in negative model.

\begin{tabular}{|c|c|c|c|c|c|}
\hline \multirow{2}{*}{ Differential metabolites } & \multirow{2}{*}{$\begin{array}{l}\text { Molecular } \\
\text { Weight }\end{array}$} & \multirow{2}{*}{$\begin{array}{l}\text { RT } \\
(\min )\end{array}$} & \multicolumn{3}{|c|}{$\log _{2}($ FoldChange $)$} \\
\hline & & & AvS Z & B vS Z & $\mathrm{C} v \mathrm{~S} \mathrm{Z}$ \\
\hline 2-Dechloroethylifosfamide & 198.03 & 4.19 & -0.47 & -0.43 & -0.40 \\
\hline 2-Hydroxyestradiol-3-methylether & 302.19 & 13.30 & -1.52 & -1.69 & -1.82 \\
\hline 3-Bromotyrosine & 258.98 & 8.68 & 6.52 & 6.60 & 6.72 \\
\hline 4-fumarylacetoacetic acid & 200.03 & 4.19 & -0.53 & -0.51 & -0.52 \\
\hline 4-Guanidinobutanoic acid & 145.09 & 0.63 & 1.92 & 1.71 & 1.79 \\
\hline 7-methylguanosine 5'-phosphate & 378.08 & 13.43 & & & 0.56 \\
\hline 9-HETE & 320.24 & 11.55 & 1.30 & & \\
\hline Adenosine tetraphosphate & 586.96 & 0.75 & 2.02 & 2.02 & \\
\hline Adenylylselenate & 474.96 & 11.66 & 7.49 & 7.72 & 8.04 \\
\hline Arachidonic Acid & 304.24 & 13.89 & -2.36 & -2.77 & -2.93 \\
\hline Arsenous acid & 125.93 & 14.81 & 16.59 & 16.82 & 17.04 \\
\hline Chondroitin & 379.11 & 8.86 & & 7.32 & 8.99 \\
\hline Cis-zeatin-7-N-glucoside & 381.16 & 14.94 & -0.88 & -1.04 & -0.91 \\
\hline $\mathrm{CPA}(18: 0 / 0: 0)$ & 420.26 & 9.97 & -1.08 & -0.93 & -0.92 \\
\hline Cytidine 2',3'-cyclic phosphate & 305.04 & 1.58 & 5.09 & 5.26 & 4.89 \\
\hline Cytidine diphosphate & 403.02 & 0.70 & & 2.66 & \\
\hline Decanoylcarnitine & 315.24 & 13.65 & & -1.63 & -1.89 \\
\hline $\operatorname{DG}(15: 0 / 16: 0 / 0: 0)$ & 554.49 & 13.78 & 14.06 & 14.46 & 14.37 \\
\hline DG(22:6(4Z,7Z,10Z,13Z,16Z,19Z)/22:2(13Z,16Z)/0:0) & 720.57 & 14.05 & 5.02 & 5.06 & 4.99 \\
\hline Diethylphosphate & 154.04 & 0.65 & 1.16 & & 1.13 \\
\hline Dimorphecolic acid & 296.24 & 12.13 & 3.00 & & \\
\hline Docosahexaenoic acid & 328.24 & 13.70 & -2.88 & -3.17 & -3.32 \\
\hline Docosapentaenoic acid & 330.26 & 14.07 & -4.50 & -4.86 & -5.16 \\
\hline Glucosylgalactosyl hydroxylysine & 486.21 & 13.33 & 3.85 & 3.61 & 3.84 \\
\hline
\end{tabular}


Table S8. Continued.

\begin{tabular}{|c|c|c|c|c|c|}
\hline Guanidineacetic acid & 117.05 & 2.29 & 4.43 & & 5.28 \\
\hline Guanosine monophosphate & 363.06 & 1.80 & 2.20 & 2.40 & 1.88 \\
\hline Hyaluronic acid & 425.15 & 13.71 & 1.43 & 1.51 & 1.49 \\
\hline Itaconyl-CoA & 879.13 & 13.00 & 13.06 & & 12.00 \\
\hline L-Cystine & 240.02 & 6.62 & -0.71 & -0.69 & -0.63 \\
\hline L-Histidine & 155.07 & 6.64 & 1.96 & 2.00 & 1.93 \\
\hline Linoleic acid & 280.24 & 14.08 & -4.91 & -5.20 & -5.21 \\
\hline L-Isoleucine & 131.09 & 1.27 & 6.07 & 5.76 & 5.72 \\
\hline $\operatorname{LPA}(0: 0 / 16: 0)$ & 410.24 & 13.45 & 1.98 & 1.96 & 1.85 \\
\hline L-Phosphoarginine & 254.08 & 3.30 & 8.13 & 7.94 & 8.14 \\
\hline L-Rhamnulose & 164.07 & 2.30 & 1.00 & & \\
\hline Melanin & 318.06 & 1.58 & 7.36 & 7.41 & 7.13 \\
\hline N2-Methylguanine & 165.07 & 2.30 & 1.10 & & \\
\hline N-Acetyl-D-glucosamine & 221.09 & 8.74 & -0.94 & -1.16 & -1.63 \\
\hline Nutriacholic acid & 390.28 & 14.40 & 5.56 & 5.07 & \\
\hline Oleic Acid & 282.26 & 14.95 & -6.11 & -6.29 & -6.31 \\
\hline Palmitic acid & 256.24 & 14.43 & -9.10 & -9.74 & down $^{\mathrm{a}}$ \\
\hline Palmitoleic acid & 254.22 & 13.82 & -2.90 & -3.14 & -3.24 \\
\hline Pantothenic Acid & 219.11 & 3.30 & 3.94 & 3.70 & 3.91 \\
\hline PC(14:0/20:4(5Z,8Z,11Z,14Z)) & 753.53 & 13.85 & down & $\mathrm{up}^{\mathrm{b}}$ & up \\
\hline PC(14:0/P-18:1(11Z)) & 715.55 & 13.26 & 3.43 & 3.44 & 3.38 \\
\hline $\mathrm{PC}(15: 0 / 16: 0)$ & 719.55 & 14.05 & 8.07 & 8.22 & 8.13 \\
\hline $\operatorname{PC}(15: 0 / 18: 0)$ & 747.58 & 14.88 & 4.79 & 5.28 & 5.22 \\
\hline $\mathrm{PC}(15: 0 / 22: 6(4 \mathrm{Z}, 7 \mathrm{Z}, 10 \mathrm{Z}, 13 \mathrm{Z}, 16 \mathrm{Z}, 19 \mathrm{Z}))$ & 791.55 & 14.86 & & 11.74 & 12.06 \\
\hline PC(18:0/22:6(4Z,7Z,10Z,13Z,16Z,19Z)) & 833.59 & 14.81 & down & up & up \\
\hline PC(20:0/22:6(4Z,7Z,10Z,13Z,16Z,19Z)) & 861.62 & 14.78 & down & up & up \\
\hline $\mathrm{PC}(20: 0 / \mathrm{P}-18: 1(11 \mathrm{Z}))$ & 799.65 & 14.86 & down & up & up \\
\hline PE(18:2(9Z,12Z)/22:6(4Z,7Z,10Z,13Z,16Z,19Z)) & 787.52 & 14.84 & & up & up \\
\hline PE(18:3(6Z,9Z,12Z)/20:5(5Z,8Z,11Z,14Z,17Z)) & 759.48 & 12.48 & 3.30 & & 3.04 \\
\hline PE(20:2(11Z,14Z)/22:6(4Z,7Z,10Z,13Z,16Z,19Z)) & 815.55 & 13.33 & 8.08 & 8.19 & 8.25 \\
\hline PE(22:5(4Z,7Z,10Z,13Z,16Z)/22:6(4Z,7Z,10Z,13Z,16Z,19Z)) & 837.53 & 13.85 & & up & \\
\hline PG(18:0/18:2(9Z,12Z)) & 774.54 & 14.86 & down & up & up \\
\hline Phosphatidylserine & 385.11 & 8.86 & & 6.22 & 7.88 \\
\hline Phosphocholine & 183.07 & 5.82 & 0.82 & 0.86 & 0.85 \\
\hline $\mathrm{PI}(16: 0 / 18: 1(11 Z))$ & 836.54 & 13.85 & & 9.95 & \\
\hline PI(16:0/20:4(5Z,8Z,11Z,14Z)) & 858.53 & 14.86 & & up & up \\
\hline PI(16:0/22:4(10Z,13Z,16Z,19Z)) & 886.56 & 14.85 & & up & up \\
\hline PI(16:0/22:5(4Z,7Z,10Z,13Z,16Z)) & 884.54 & 14.85 & down & up & up \\
\hline PI(18:0/22:5(4Z,7Z,10Z,13Z,16Z)) & 912.57 & 14.84 & down & up & up \\
\hline Pregnenolone & 316.24 & 13.65 & -1.40 & -1.56 & -1.77 \\
\hline $\operatorname{PS}(16: 0 / 16: 0)$ & 735.51 & 13.85 & 9.25 & 9.12 & 8.98 \\
\hline
\end{tabular}


Table S8. Continued.

\begin{tabular}{|c|c|c|c|c|c|}
\hline S-Adenosylhomocysteine & 384.12 & 12.99 & 2.78 & 2.74 & 2.63 \\
\hline SM(d17:1/24:1(15Z)) & 798.66 & 14.86 & down & up & up \\
\hline Tauroursodeoxycholic acid & 499.30 & 9.94 & 4.09 & 3.96 & 3.58 \\
\hline TG(18:3(9Z,12Z,15Z)/14:0/18:3(9Z,12Z,15Z)) & 822.67 & 13.98 & down & up & up \\
\hline trifluoroacetic acid & 113.99 & 0.65 & 0.89 & & \\
\hline Uridine 5'-monophosphate & 324.04 & 1.16 & -1.50 & -1.48 & -1.81 \\
\hline
\end{tabular}

${ }^{\text {a }}$ Down means that the treatment group is 0 , and the metabolite is detected in the group Z;

${ }^{\mathrm{b}} \mathrm{Up}$ means that the measured value of group $\mathrm{Z}$ is 0 , and the metabolite is detected in the treatment group.

a)

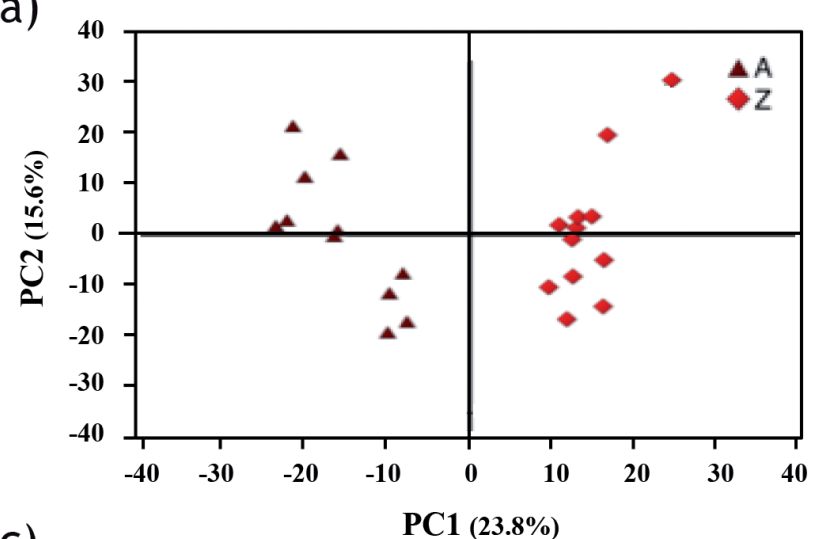

C)

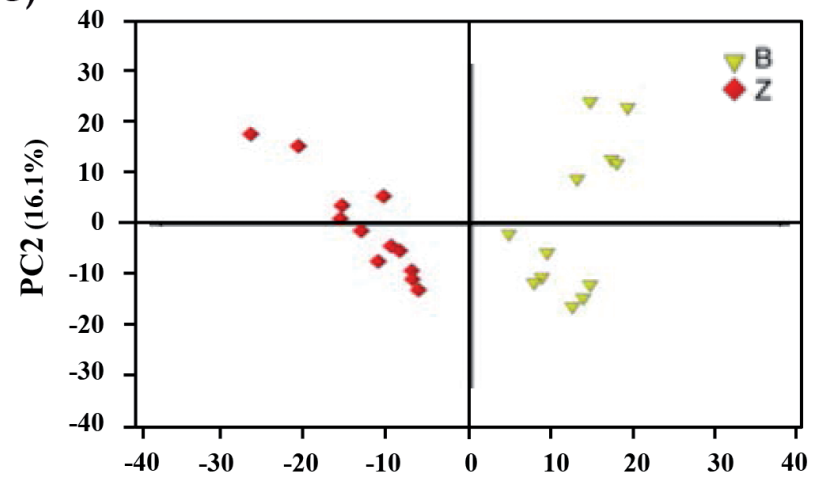

e)

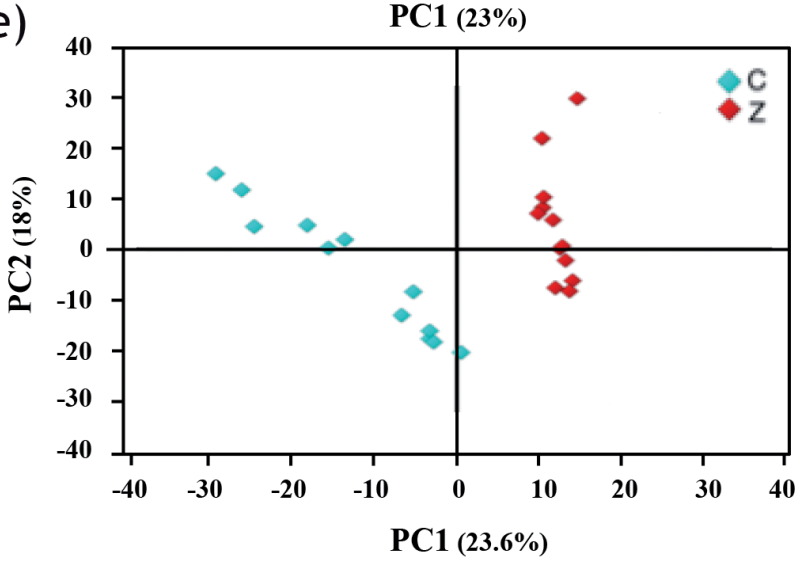

b)

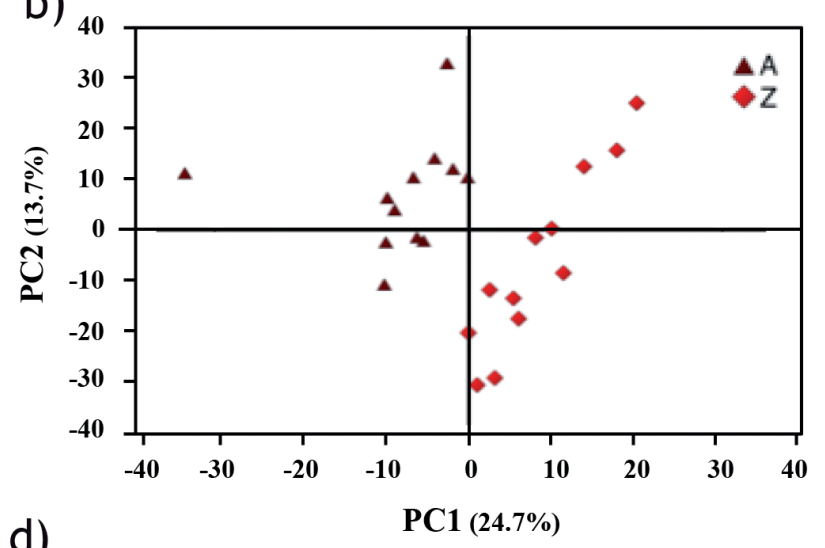

d)

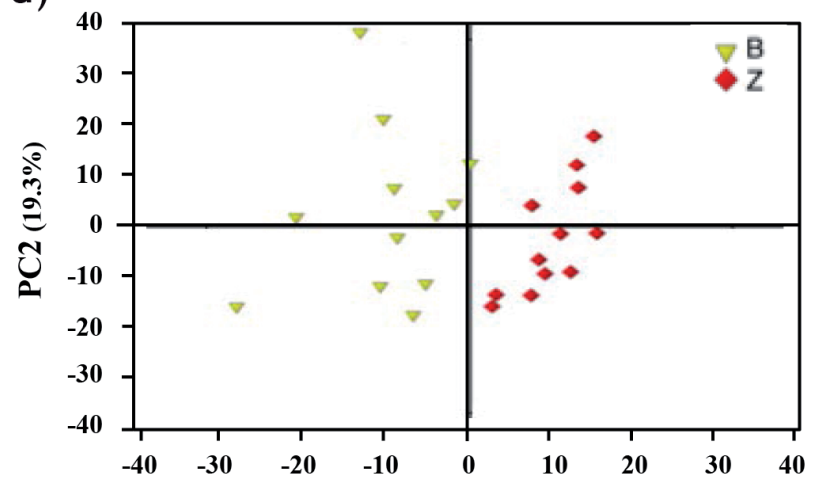

f)

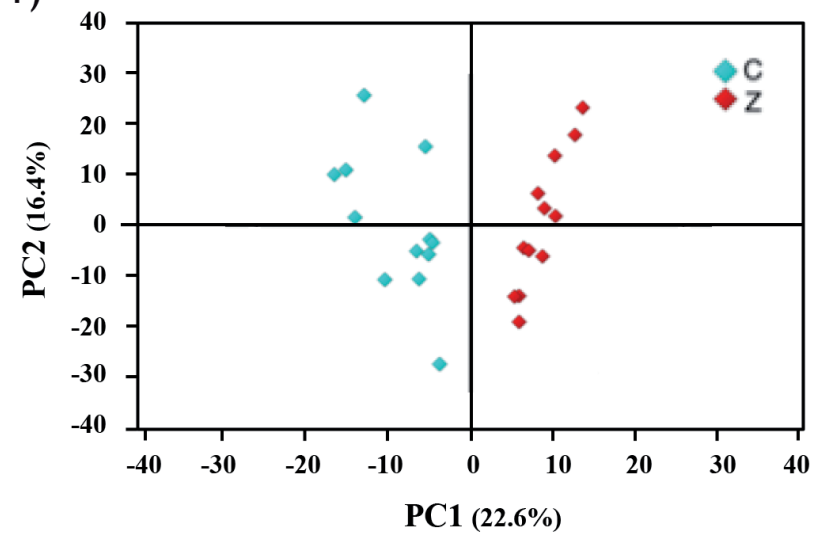

Fig. S1. The PCA score plots of serum samples A-Z (A: ESI+, B: ESI-), B-Z (C: ESI+ , D: ESI') and C-Z (E: ESI+ ${ }^{+}$F: ESI-).a), c) and e). The scoring plot of serum samples A, B, C and Z by comparing the contents of a total of 812 features extracted in positive ion mode. PC1 and $\mathrm{PC} 2$ of A-Z, B-Z and C-Z described $23.8 \%$ and $15.6 \%, 23 \%$ and $16.1 \%, 23.6$ and $18 \%$ of total variability, respectively. b), d) and f). The scoring plot of serum samples A, B, C and Z by comparing the contents of a total of 554 features extracted in negative ion mode. PC1 and PC2 of A-Z, B-Z and C-Z described $24.7 \%$ and $13.7 \%, 24 \%$ and $19.3 \%, 22.6 \%$ and $16.4 \%$ of total variability, respectively. 
a)

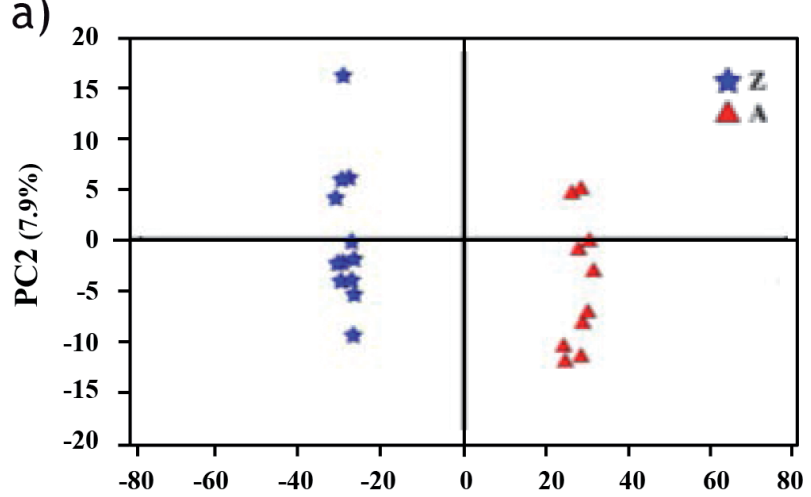

c)
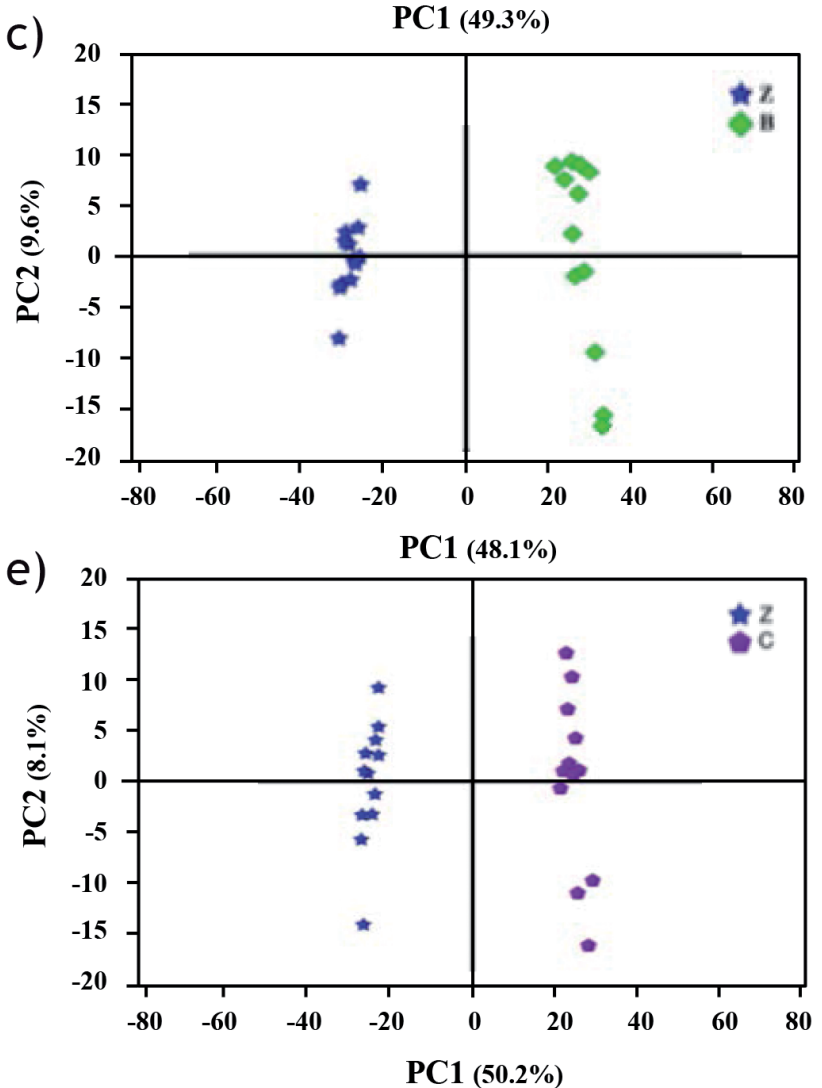

b)

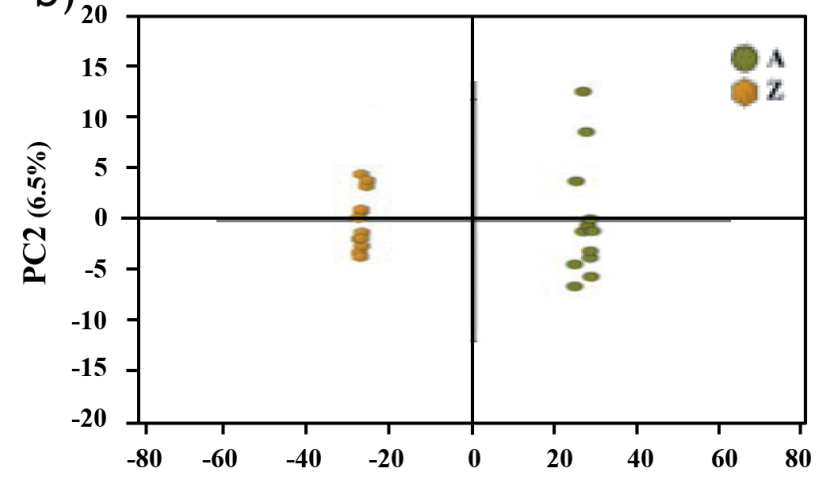

d)
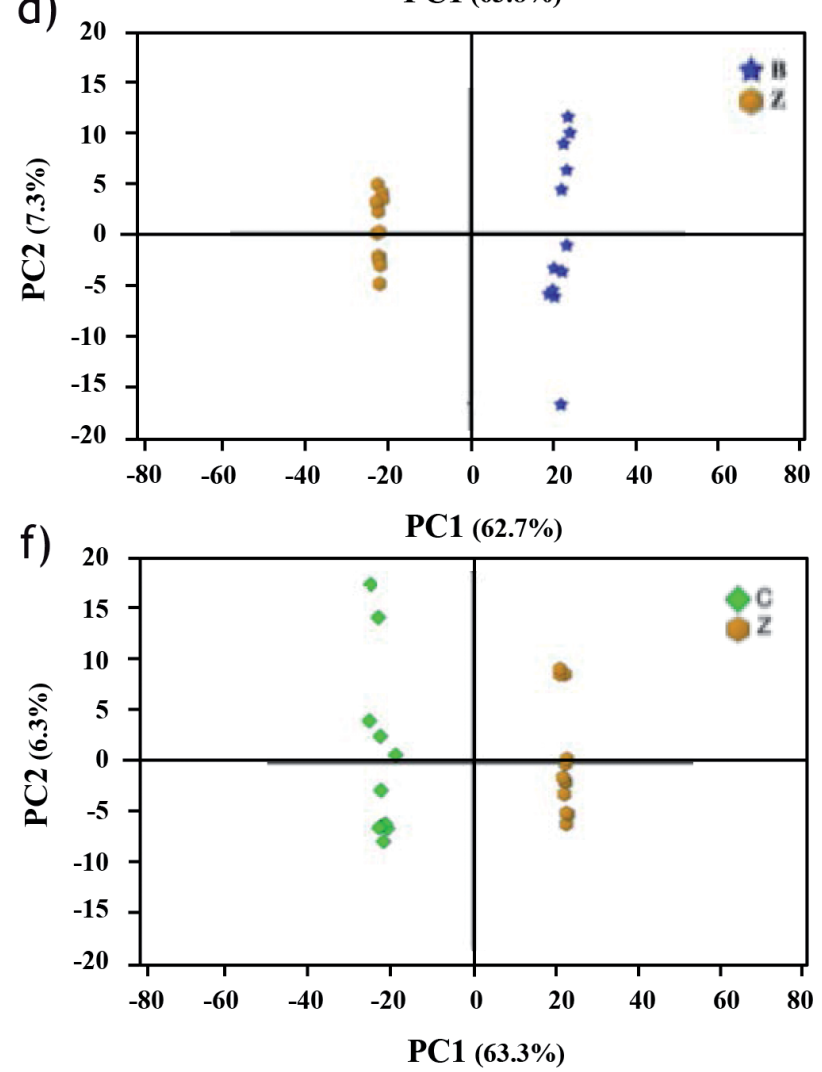

Fig. S2. The PCA score plots of liver samples A-Z (A: ESI+ ${ }^{+}$B: ESI-), B-Z (C: ESI $\left.{ }^{+}, D^{-}: E^{-}\right)$and C-Z (E: ESI', F: ESI-). a), c) and e). The scoring plot of serum samples $A, B, C$ and $Z$ by comparing the contents of a total of 1,391 features extracted in positive ion mode. PC1 and PC2 of A-Z, B-Z and C-Z described $49.3 \%$ and $7.9 \%, 48.1 \%$ and $9.6 \%, 50.2$ and $8.1 \%$ of total variability, respectively. b), d) and $\mathrm{f}$ ). The scoring plot of serum samples $\mathrm{A}, \mathrm{B}, \mathrm{C}$ and $\mathrm{Z}$ by comparing the contents of a total of 1,116 features extracted in negative ion mode. PC1 and PC2 of A-Z, B-Z and C-Z described $63.8 \%$ and $6.5 \%, 62.7 \%$ and $7.3 \%, 63.3 \%$ and $6.3 \%$ of total variability, respectively. 


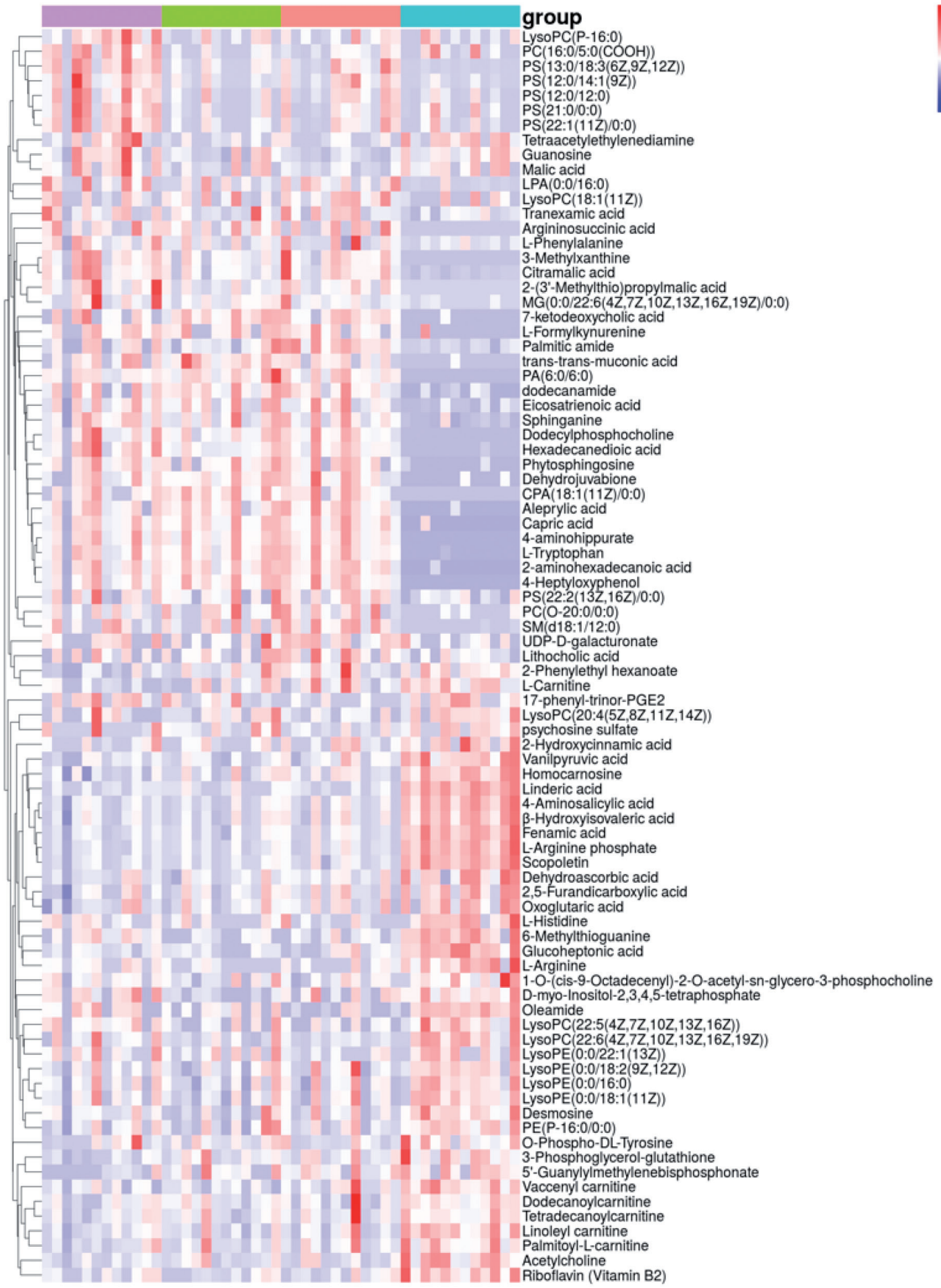

Fig. S3. Clustering analysis of metabolomic data in 4 groups of serum samples $\left(\mathrm{ESI}^{+}\right)$. A heat map representation of the levels of 87 metabolites in $\mathrm{ESI}^{+}$mode. Each line in the heat map represents a metabolite while each file represents a sample. Red indicates concentration of metabolite levels greater than that of median, and blue indicates concentration of metabolite levels lower than that of median. 


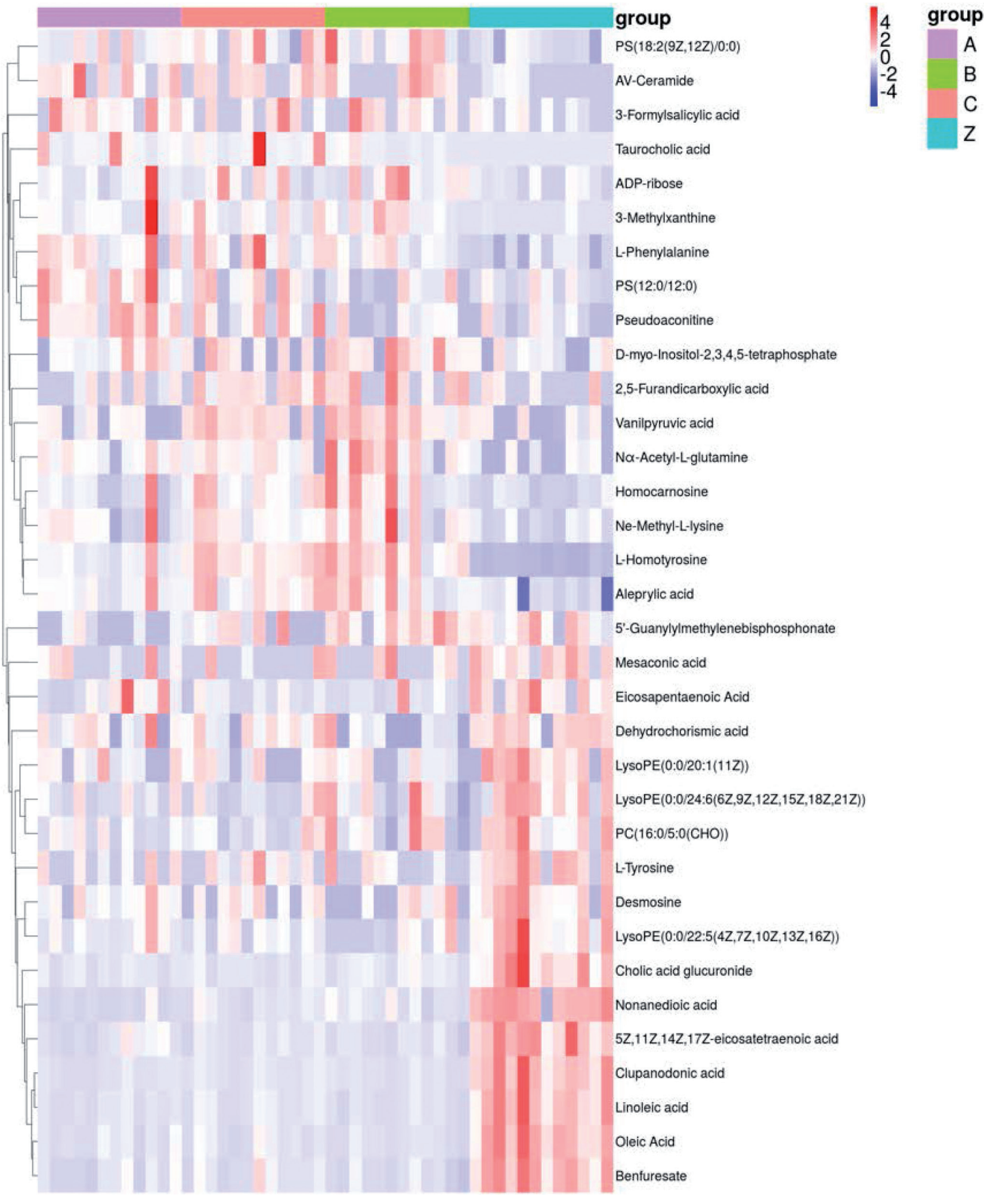

Fig. S4. Clustering analysis of metabolomic data in 4 groups of serum samples (ESI). A heat map representation of the levels of 34 metabolites in ESI- mode. Each line in the heat map represents a metabolite while each file represents a sample. Red indicates relative expression of metabolite levels greater than that of median, and blue indicates relative expression of metabolite levels lower than that of median. 

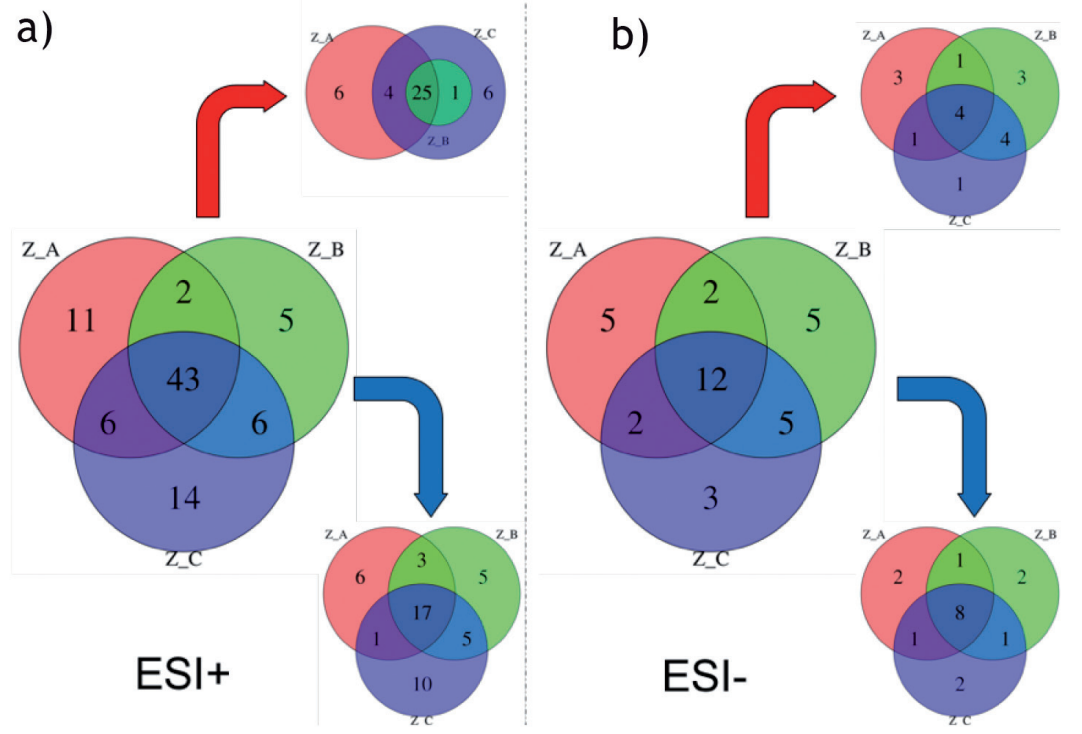

Fig. S5. Venn diagram of metabolomic data from 3 comparisons (A-Z, B-Z, and C-Z) of the serum samples. Venn diagrams (a) ESI b) ESI-) represent quantitative relationship of differential metabolites among 3 comparisons. Each figure in the Venn diagram represents the metabolite number of corresponding aspect. Red arrow indicates up-regulation of relative expression of metabolites while the venn diagram of its point reveals quantitative relationship of up-regulation metabolites, and blue arrow indicates down-regulation of relative expression of metabolites while the venn diagram of its point reveals quantitative relationship of down-regulation metabolites. 


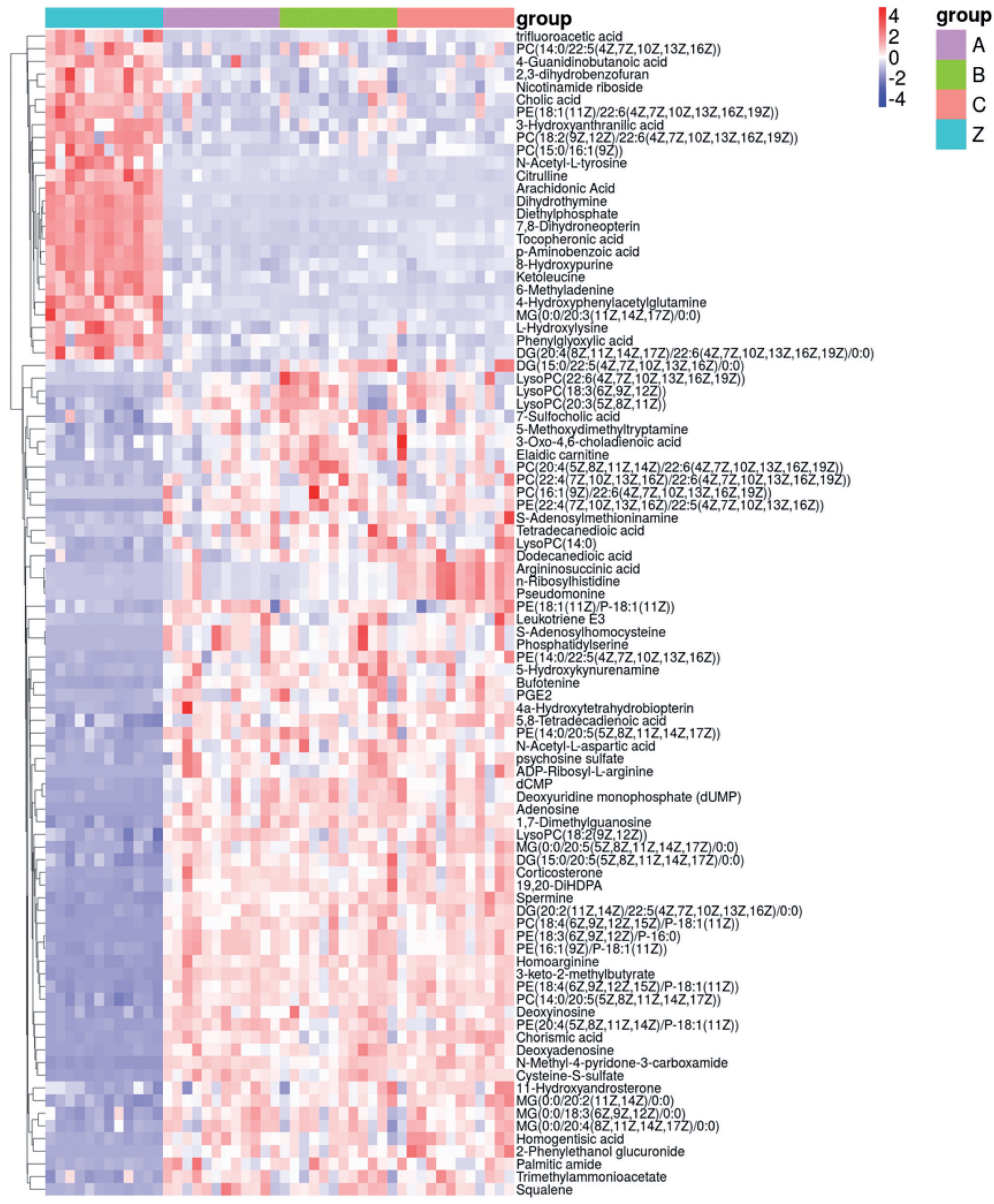

Fig. S6. Clustering analysis of metabolomic data in 4 groups of liver samples $\left(\mathrm{ESI}^{+}\right)$. A heat map representation of the levels of 92 metabolites in $\mathrm{ESI}^{+}$mode. Each line in the heat map represents a metabolite while each file represents a sample. Red indicates concentration of metabolite levels greater than that of median, and blue indicates concentration of metabolite levels lower than that of median. 


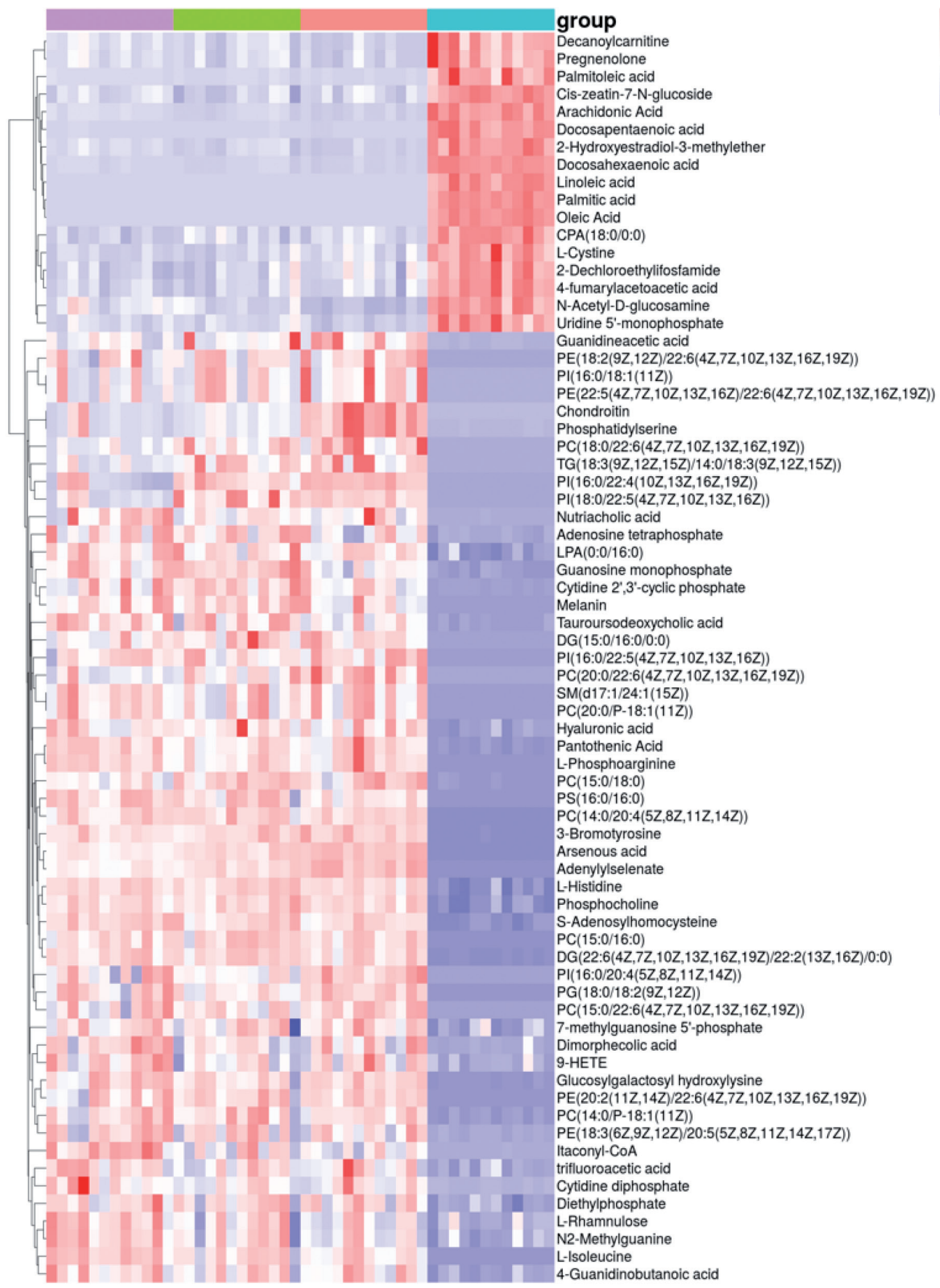

Fig. S7. Clustering analysis of metabolomic data in 4 groups of liver samples (ESI-). A heat map representation of the levels of 71 metabolites in ESI- mode. Each line in the heat map represents a metabolite while each file represents a sample. Red indicates relative expression of metabolite levels greater than that of median, and blue indicates relative expression of metabolite levels lower than that of median. 

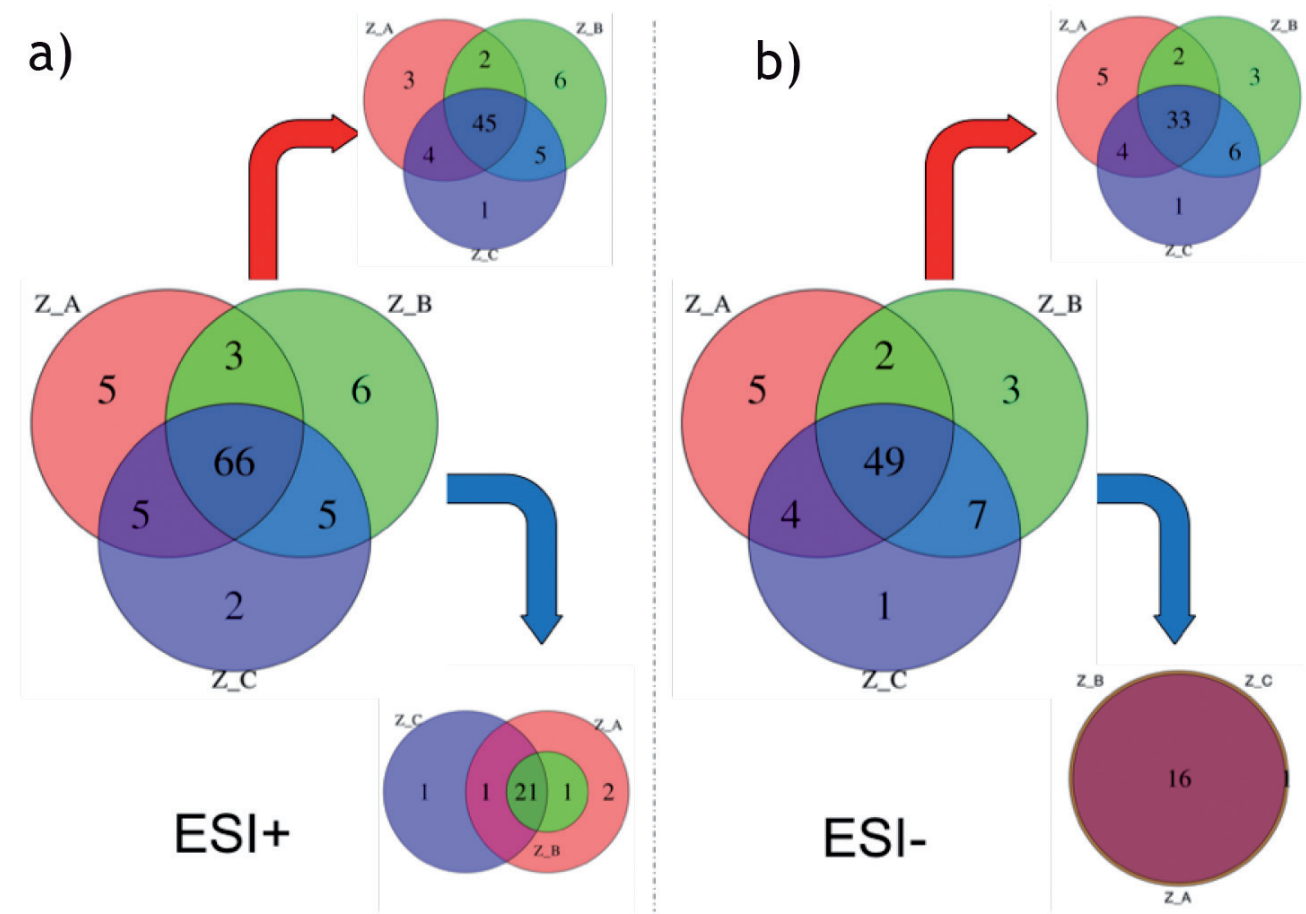

Fig. S8. Venn diagram of metabolomic data from 3 comparisons (A-Z, B-Z, and C-Z) of the liver samples. Venn diagrams (a) ESI ${ }^{+}$, b)

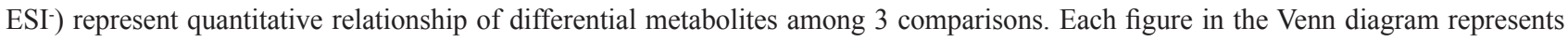
the metabolite number of corresponding aspect. Red arrow indicates up-regulation of relative expression of metabolites while the venn diagram of its point reveals quantitative relationship of up-regulation metabolites, and blue arrow indicates down-regulation of relative expression of metabolites while the venn diagram of its point reveals quantitative relationship of down-regulation metabolites. 